\title{
Exact Solutions of Diffusion-Convection Equations
}

\author{
Nataliya M. Ivanova
}

Communicated by Y. Charles Li, received October 31, $200 \%$.

\begin{abstract}
In the presented paper known (up to the beginning of 2008) Lieand non-Lie exact solutions of different $(1+1)$-dimensional diffusion-convection equations of form $f(x) u_{t}=\left(g(x) A(u) u_{x}\right)_{x}+h(x) B(u) u_{x}$ are collected.
\end{abstract}

\section{Contents}

1. Introduction 139

2. Linear heat equation 140

3. Linearizable equations $\quad 142$

4. Nonlinear diffusion equations. General case 144

5. Nonlinear diffusion equations. Exponential nonlinearity 145

6. Nonlinear diffusion equations. Power nonlinearities 146

7. Porous medium equation $\boldsymbol{u}_{\boldsymbol{t}}=\left(\boldsymbol{u} \boldsymbol{u}_{\boldsymbol{x}}\right)_{\boldsymbol{x}} \quad 148$

8. Fast diffusion equation $\boldsymbol{u}_{\boldsymbol{t}}=\left(\boldsymbol{u}^{-\mathbf{1}} \boldsymbol{u}_{\boldsymbol{x}}\right)_{\boldsymbol{x}} \quad 149$

9. Nonlinear diffusion equations. Other nonlinearities 151

10. Constant coefficient diffusion-convection equations 154

11. $\boldsymbol{n}$-dimensional radially symmetric nonlinear

12. Variable coefficient diffusion-convection equations 158

13. Examples of Lie reduction of variable coefficient equation 162

14. Exact solutions of $\boldsymbol{s l}(\mathbf{2}, \mathbb{R})$-invariant equation 164

References $\quad 165$

Appendix A. Group classification of diffusion-convection equations $\quad 169$

\section{Introduction}

This is a review paper, where we present a brief summary of known exact solutions of variable coefficient $(1+1)$-dimensional diffusion-convection equations

1991 Mathematics Subject Classification. 35.

Key words and phrases. Diffusion-convection equations, Lie- and non-Lie exact solutions.

(C)2008 International Press 
of form

$$
f(x) u_{t}=\left(g(x) A(u) u_{x}\right)_{x}+h(x) B(u) u_{x},
$$

where $f=f(x), g=g(x), h=h(x), A=A(u)$ and $B=B(u)$ are arbitrary smooth functions of their variables, $f(x) g(x) A(u) \neq 0$.

Our aim is not to give a physical interpretation of the solution of diffusion equations (that is too huge and cannot be reached in the scope of a short paper), but to list the already known exact solutions of equations from the class under consideration. However, in some cases we give a short discussion of the nature of the listed solutions.

The majority of the listed solutions have been obtained by means of different symmetry methods, such as reduction with respect to Lie and non-Lie symmetries, separation of variables, equivalence transformations, etc.

Let us note that the constant coefficient diffusion equations $(f=g=1, B=0)$ are well investigated and some of exact solutions given below were summarized before in $[\mathbf{2 6}, \mathbf{4 8}]$.

Our paper is organized as follows. First of all we adduce solutions of the linear heat equation obtained by means of various symmetry methods. In Section 3 the linearizable Burgers, Fujita-Storm and Fokas-Yortsos equations are considered. Lie reduction of constant coefficient nonlinear diffusion equation $(h B=0, f=g=1)$ is performed in Section 4. Solutions of constant coefficient diffusion equations with exponential nonlinearity are adduced in Section 5. Solutions of constant coefficient diffusion equations with power nonlinearity are presented in Section 6. The important particular case of such equations, namely, the fast diffusion equation, is studied in more detail in Section 8. Diffusion equations with other nonlinearities are briefly discussed in Section 9. The next considered case (Section 10) covers the nonlinear constant coefficient diffusion-convection equations $(f=g=h=1)$. In Section 11 we adduce a brief analysis of known solutions of $n$-dimensional radially symmetric nonlinear diffusion equations. In Section 12 exact solutions of some variable coefficient diffusion-convection equations are collected. At last, in Sections 13 and 14 we present a detailed analysis of interesting variable coefficient equations having distinguished invariance properties.

In the Appendix A we adduce the complete results of group classification of equations (1) with respect to the extended group $\hat{G}^{\sim}$ of equivalence transformations (23).

Below, if it is not indicated separately, $\alpha, \varepsilon_{i}, \lambda, a, b, c, c_{i}$ are arbitrary constants, $\varepsilon= \pm 1$. For convenience we use double numeration T.N of classification cases and local equivalence transformations, where $\mathrm{T}$ denotes the number of table and $\mathrm{N}$ does the number of case (or transformation, or solution) in table T. The notion "equation T.N" is used for the equation of form (1) where the parameter-functions $f, g, h, A, B$ take values from the corresponding case.

\section{Linear heat equation}

Systematical investigation of invariant solutions of different diffusion equations was started by the case of linear heat equation $[\mathbf{3 2}, 33,39,42,44,45]$

$$
u_{t}=u_{x x}
$$


which is invariant with respect to the six dimensional symmetry algebra generated by the vector fields

$$
\begin{gathered}
Q_{1}=\partial_{x}, \quad Q_{2}=\partial_{t}, \quad Q_{3}=u \partial_{u}, \quad Q_{4}=2 t \partial_{t}+x \partial_{x} \\
Q_{5}=2 t \partial_{x}-x u \partial_{u}, \quad Q_{6}=4 t^{2} \partial_{t}+4 t x \partial_{x}-\left(x^{2}+2 t\right) u \partial_{u}
\end{gathered}
$$

(For the moment we are ignoring the trivial infinite-dimensional subalgebras coming from the linearity of the heat equation and corresponding to the linear superposition principle).

The most general solution obtainable from a given solution $u=f(t, x)$ by group transformations is of the form

(3) $\tilde{u}=\frac{\varepsilon_{3}}{\sqrt{1+4 \varepsilon_{6} t}} e^{-\frac{\varepsilon_{5} x+\varepsilon_{6} x^{2}-\varepsilon_{5}^{2} t}{1+4 \varepsilon_{6} t}} f\left(\frac{\varepsilon_{4}^{2} t}{1+4 \varepsilon_{6} t}-\varepsilon_{2}, \frac{\varepsilon_{4}\left(x-2 \varepsilon_{5} t\right)}{1+4 \varepsilon_{6} t}-\varepsilon_{1}\right)+v(t, x)$,

where $v(t, x)$ is an arbitrary solution to the linear heat equation [42]. Considering the higher-order symmetry generators, one can prove that if $u=f(t, x)$ is a solution of the linear heat equation than

$$
\begin{gathered}
\tilde{u}=2 t f_{x}(t, x)+x f(t, x) \text { and } \\
\tilde{u}=t^{2} f_{t}(t, x)+t x f_{x}(t, x)+\frac{1}{4}\left(2 t+x^{2}\right) f(t, x)
\end{gathered}
$$

are also solutions of the same equation [4].

All possible inequivalent (with respect to inner automorphisms) one-dimensional subalgebras of the given algebra are exhausted by the ones listed in Table 1 [68] together with the corresponding ansatzes and the reduced ODEs.

Table 1. Reduced ODEs for linear heat equation (2)

\begin{tabular}{|c|l|c|c|l|}
\hline $\mathrm{N}$ & Subalgebra & Ansatz $u=$ & $\omega$ & Reduced ODE \\
\hline 1 & $\left\langle Q_{4}+a Q_{3}\right\rangle$ & $t^{a} \varphi(\omega)$ & $x / \sqrt{t}$ & $\varphi^{\prime \prime}+\frac{\omega \varphi^{\prime}}{2}-a \varphi=0$ \\
2 & $\left\langle Q_{2}+Q_{6}+a Q_{3}\right\rangle$ & $\left(4 t^{2}+1\right)^{\frac{1}{4}} \varphi(\omega)$ & $\left(4 t^{2}+1\right)^{-\frac{1}{2}} x$ & $\varphi^{\prime \prime}+\left(a+\omega^{2}\right) \varphi=0$ \\
& & $e^{-\left(4 t^{2}+1\right)^{-1} t x^{2}-a \arctan (2 t) / 2}$ & & \\
3 & $\left\langle Q_{2}-Q_{5}\right\rangle$ & $\varphi(\omega) e^{t x+2 t^{3} / 3}$ & $x+t^{2}$ & $\varphi^{\prime \prime}=\omega \varphi$ \\
4 & $\left\langle Q_{2}+a Q_{3}\right\rangle$ & $\varphi(\omega) e^{a t}$ & $x$ & $\varphi^{\prime \prime}=a \varphi$ \\
5 & $\left\langle Q_{1}\right\rangle$ & $\varphi(\omega)$ & $t$ & $\varphi^{\prime}=0$ \\
6 & $\left\langle Q_{3}\right\rangle$ & - & - & \\
\hline
\end{tabular}

Thus we have the following solutions of (2):

$$
\begin{gathered}
u=t^{a} e^{-x^{2} /(8 t)}\left(c_{1} U\left(2 a+\frac{1}{2}, \frac{x}{\sqrt{2 t}}\right)+c_{2} V\left(2 a+\frac{1}{2}, \frac{x}{\sqrt{2 t}}\right)\right), \\
u=\left(4 t^{2}+1\right)^{1 / 4}\left(c_{1} W\left(-\frac{a}{2}, \frac{x}{\sqrt{8 t^{2}+2}}\right)\right. \\
\left.+c_{2} W\left(-\frac{a}{2},-\frac{x}{\sqrt{8 t^{2}+2}}\right)\right) e^{-\frac{t x^{2}}{\left(4 t^{2}+1\right)}-\frac{a}{2} \arctan (2 t)}, \\
u=c_{1} e^{\alpha^{2} t} \cosh \left(\alpha x+c_{2}\right), \quad u=c_{1} x+c_{2}, \quad u=c_{1} e^{-\alpha^{2} t} \cos \left(\alpha x+c_{2}\right),
\end{gathered}
$$

where $U(b, z), V(b, z), W(c, z)$ are parabolic cylinder functions [1].

Multiplicative separation of variables leads to the solutions invariant with respect to $\left\langle Q_{2}+a Q_{3}\right\rangle$. The additive separation of variables yields an exact solution of form

$$
u=c_{1} x^{2}+c_{2} x+2 c_{1} t
$$


The known $Q$-conditional symmetry operators and corresponding reductions are adduced in Table 2. [21]

Table 2. Nonclassical reductions for linear heat equation (2)

\begin{tabular}{|c|c|c|c|}
\hline $\mathrm{N}$ & Operator $Q$ & Ansatz & Reduced equation \\
\hline 1 & $-x \partial_{t}+\partial_{x}$ & $u=\varphi\left(t+\frac{x^{2}}{2}\right)$ & $\varphi^{\prime \prime}=0$ \\
2 & $-x \partial_{t}+\partial_{x}+x^{3} \partial_{u}$ & $u=\varphi\left(t+\frac{x^{2}}{2}\right)+\frac{x^{4}}{4}$ & $\varphi^{\prime \prime}=-3$ \\
3 & $x^{2} \partial_{t}-3 x \partial_{x}-3 u \partial_{u}$ & $u=x \varphi\left(t+\frac{x^{2}}{6}\right)$ & $\varphi^{\prime \prime}=0$ \\
4 & $x^{2} \partial_{t}-3 x \partial_{x}-\left(3 u+x^{5}\right) \partial_{u}$ & $u=x \varphi\left(t+\frac{x^{2}}{6}\right)+\frac{x^{5}}{12}$ & $\varphi^{\prime \prime}=-15$ \\
5 & $x \partial_{x}+u \partial_{u}$ & $u=x \varphi(t)$ & $\varphi^{\prime}=0$ \\
6 & $\operatorname{coth} x \partial_{x}+u \partial_{u}$ & $u=\varphi(t) \cosh x$ & $\varphi^{\prime}-\varphi=0$ \\
7 & $-\cot x \partial_{x}+u \partial_{u}$ & $u=\varphi(t) \cos x$ & $\varphi^{\prime}+\varphi=0$ \\
8 & $\partial_{x}-u \partial_{u}-\frac{u}{2 t-x} \partial_{u}$ & $u=(2 t-x) e^{-x} \varphi(t)$ & $\varphi^{\prime}-\varphi=0$ \\
9 & $\partial_{x}-\sqrt{-2(t+u)} \partial_{u}$ & $u=-t-\frac{1}{2}[x+\varphi(t)]^{2}$ & $\varphi^{\prime}=0$ \\
10 & $\left(t+\frac{x^{2}}{2}\right) \partial_{t}-x \partial_{x}$ & $u=\varphi\left(t x+\frac{x^{2}}{3 !}\right)$ & $\varphi^{\prime \prime}=0$ \\
\hline
\end{tabular}

If function $f(t, x)$ is an arbitrary solution of the linear heat equation and $u$ is the general integral of the ODE $f_{x} d t+f d x=0$, then $u$ satisfies the linear heat equation. This statement can be considered as another algorithm of generating solutions of the linear heat equation [21]. Indeed, even starting from a rather trivial solution of the heat equation $u=1$ one gets the chain of quite interesting solutions

$$
1 \rightarrow x \rightarrow t+\frac{x^{2}}{2 !} \rightarrow t x+\frac{x^{3}}{3 !} \rightarrow \cdots,
$$

and among them the solutions

$$
\begin{gathered}
\frac{x^{2 m}}{(2 m) !}+\frac{t}{1 !} \frac{x^{2 m-2}}{(2 m-2) !}+\frac{t^{2}}{2 !} \frac{x^{2 m-4}}{(2 m-4) !}+\cdots+\frac{t^{m-1}}{(m-1) !} \frac{x^{2}}{2 !}+\frac{t^{m}}{m !}, \\
\frac{x^{2 m+1}}{(2 m+1) !}+\frac{t}{1 !} \frac{x^{2 m-1}}{(2 m-1) !}+\frac{t^{2}}{2 !} \frac{x^{2 m-3}}{(2 m-3) !}+\cdots+\frac{t^{m-1}}{(m-1) !} \frac{x^{3}}{3 !}+\frac{t^{m}}{m !} \frac{x}{1 !},
\end{gathered}
$$

called often the heat polynomials $[\mathbf{6 9}]$.

\section{Linearizable equations}

Class (1) contains three equations, namely Burgers equation

$$
u_{t}=u_{x x}+2 u u_{x}
$$

Fujita-Storm equation

$$
u_{t}=\left(u^{-2} u_{x}\right)_{x}
$$

and Fokas-Yortsos equation

$$
u_{t}=\left(u^{-2} u_{x}\right)_{x}+u^{-2} u_{x}
$$

that are linearizable by the potential equivalence hodograph transformation and additional local equivalence transformations $[\mathbf{1 0}, \mathbf{1 4}, \mathbf{1 8}, \mathbf{1 9}, \mathbf{2 5}, \mathbf{3 8}, \mathbf{5 0}, \mathbf{6 3}, \mathbf{6 4}]$ : 


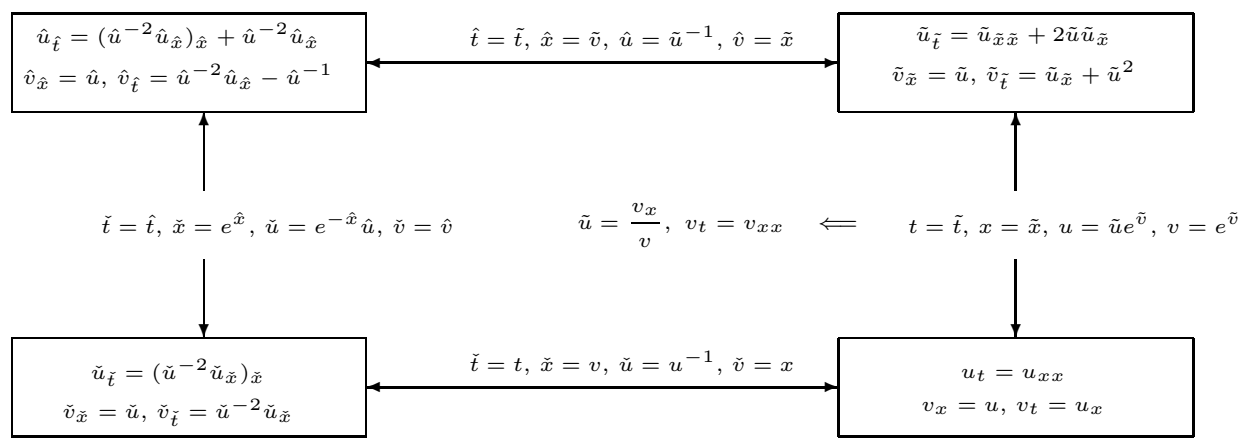

Therefore, applying the above transformations to the well-known solutions of the linear heat equation one can easily construct solutions of the linearizable equations.

Thus, e.g., the fundamental (source) solution $u=(4 \pi t)^{-1 / 2} e^{-x^{2} /(4 t)}$ and dipole solution $u=-\left((4 \pi t)^{-1 / 2} e^{-x^{2} /(4 t)}\right)_{x}$ are mapped into the separable and self-similar solutions of the Fujita-Storm equation $[48]$

$$
\begin{gathered}
u=(4 \pi t)^{1 / 2} e^{v^{2}} \quad \text { where } \quad x=\pi^{-1 / 2} \int_{0}^{v} e^{-y^{2}} d y, \\
\text { and } u=x^{-1}(2 t)^{1 / 2}\left(\ln \frac{1}{4 \pi t x^{2}}\right)^{-1 / 2} .
\end{gathered}
$$

correspondingly.

Other solutions of the linear heat equation presented in the previous section yield the following explicite exact solutions of the Fujita-Storm equation [30]:

$$
\begin{aligned}
& u=c, \quad u=x^{-1}, \quad u=(x-2 t)^{-\frac{1}{2}}, \quad u=\left(x^{2} \pm e^{2 t}\right)^{-\frac{1}{2}}, \\
& u= \pm\left(e^{-2 t}-x^{2}\right)^{-\frac{1}{2}}, u=\frac{1}{4 \sqrt{24 t^{2}+x} \sqrt{-6 t \pm \sqrt{24 t^{2}+x}}}, u=\frac{t}{x \sqrt{-t \ln (x \sqrt{t})}} \\
& u=\frac{1}{\sqrt{c_{1}^{2} e^{-2 t}+2 e^{-8 t}+2 e^{-4 t} x} \sqrt{4-e^{8 t}\left(-c_{1} e^{-t} \pm \sqrt{c_{1}^{2} e^{-2 t}+2 e^{-8 t}+2 e^{-4 t} x}\right)^{2}}}, \\
& u=\frac{1}{\sqrt{c_{1}^{2} e^{2 t}+2 e^{8 t}+2 e^{4 t} x} \sqrt{e^{-8 t}\left(-c_{1} e^{-t} \pm \sqrt{c_{1}^{2} e^{-2 t}+2 e^{-8 t}+2 e^{-4 t} x}\right)^{2}-4}} .
\end{aligned}
$$

In [66] the following formula for deriving exact solutions of the Fujita-Storm equation is derived: if $u(t, x)$ is a solution of the Fujita-Storm equation (5) then

$$
v=u+\frac{2 t u_{t}+u+x u_{x}}{-t\left(u_{x}-\frac{1}{2} u^{2}\right)-\frac{x}{2} u}
$$

is also solution of the same equation.

Similarly [67], if $u(t, x)$ is a solution of the and the Fokas-Yortsos equation (6) then

$$
v=\frac{u^{2}}{u-u_{x}}
$$


is also solution of the same equation. In [67] a new exact solution of the FokasYortsos equation is presented:

$$
u=\frac{1}{2} \frac{W\left(e^{4 t+x}\right)}{1+W\left(e^{4 t+x}\right)},
$$

where $W(x)$ is the Lambert $W$ function determined as $W(x) e^{W(x)}=x$. Let us note that other exact solutions of the Fokas-Yortsos equation (6) can be easily recovered from the solution set of the Fujita-Storm equation (5) by means of application of the local transformation of variables shown in the above scheme.

The same tricks can be used for obtaining exact solutions of the remaining linearizable equations. However, since the adduced transformations are nonlocal, sometimes it could be easier to search directly for solutions of the nonlinear equations. Thus, e.g., one can easily find Lie solutions of the Burgers equation:

$$
\begin{gathered}
u=\frac{c_{1}-x}{2 t+c_{2}}, \quad u=\varepsilon+\frac{1}{x+2 \varepsilon t+c}, \quad u=\frac{2 x+c_{1}}{x^{2}+c_{1} x+2 t+c_{2}}, \\
u=\frac{6\left(x^{2}+2 t+c_{1}\right)}{2 x^{3}+12 t x+6 c_{1} t+c_{2}}, \quad u=\frac{c_{1}}{1+c_{2} e^{-c_{1}^{2} t-c_{1} x}}, \quad u=-\varepsilon+\frac{a}{2} \frac{e^{c_{1}(x-2 \varepsilon t)}-c_{2}}{e^{c_{1}(x-2 \varepsilon t)}+c_{2}}, \\
u=-\varepsilon+c_{1} \tanh \left(c_{1}(x-2 \varepsilon t)+c_{2}\right), \\
u=\frac{\lambda}{2\left(\lambda^{2} t+c_{1}\right)}\left(2 \tanh \frac{\lambda x+c_{2}}{\lambda^{2} t+c_{1}}-\lambda x-c_{2}\right), \\
u=-\varepsilon-c_{1} \tan \left(c_{1}(x-2 \varepsilon t)+c_{2}\right), \quad u=\frac{\lambda \cos \left(\lambda x+c_{1}\right)}{c_{2} e^{\lambda^{2} t+\sin \left(\lambda x+c_{1}\right)},} \\
u=\frac{c_{1}}{\sqrt{\pi\left(t+c_{2}\right)}} \exp \left(-\frac{x+c_{3}}{4\left(t+c_{2}\right)}\right)\left(c_{1} \operatorname{erf} \frac{x+c_{3}}{2 \sqrt{t+c_{2}}}+c_{4}\right)^{-1}, \\
u=-\frac{\cos 2 x e^{-3 t}+c_{1} \sin x}{-\cos x \sin x e^{-3 t}+c_{1} \cos x+c_{2} e^{t}}, \\
u=-\frac{c_{1} e^{-t}(\cos x+\sin x)+c_{2} e^{t+x}}{c_{1} e^{-t}(\cos x-\sin x)-c_{2} e^{t+x}+b} .
\end{gathered}
$$

Here $\operatorname{erf} z=\frac{2}{\sqrt{\pi}} \int_{0}^{z} e^{-\xi^{2}} d \xi$ is the error function (also called the probability integral). The last two solutions were found in [54].

Solutions of the Fujita-Storm and Fokas-Yortsos equations can be singled out form the solutions adduced in Sections 6 and 10 taking $\mu=-2, \nu=-2$. (Note that all these solutions can be also reconstructed from ones of the linear heat equation by means of potential equivalence transformations.)

If if $u(t, x)$ is a solution of the Burgers equation (4) then

$$
v=u+\frac{u_{t}}{u_{x}+u^{2}}, \quad u=u+\frac{u_{t}+u_{x}}{u_{x}+u^{2}+u}
$$

are also solutions of the same equation $[\mathbf{6 6}]$.

Potential equivalence transformations were used to obtain solutions of some boundary-value problems adduced in $[\mathbf{1 0}, \mathbf{4 1}, \mathbf{6 3}, \mathbf{6 4}]$

\section{Nonlinear diffusion equations. General case}

Consider now the class of nonlinear diffusion equations

$$
u_{t}=\left(A(u) u_{x}\right)_{x}
$$


where $A_{u} \neq 0$. Lie symmetries of this class have been studied in [44]. The Lie symmetry algebra of equation from class (7) with arbitrary value of parameterfunction $A(u)$ is three-dimensional and spanned by

$$
Q_{1}=\partial_{t}, \quad Q_{2}=\partial_{x}, \quad Q_{3}=2 t \partial_{t}+x \partial_{x} .
$$

Taking into account discrete symmetry transformations of changing sings of independent variables one can formulate the following statement. If $u=f(t, x)$ is a solution of equation (7), then $\tilde{u}=f\left(\varepsilon_{1}^{2} t+\varepsilon_{2}, \varepsilon_{1} x+\varepsilon_{3}\right)$ is also solution of the same equation.

All possible inequivalent (with respect to inner automorphisms) one-dimensional subalgebras of the given symmetry algebra,the corresponding ansatzes and the reduced ODEs are exhausted by the ones listed in Table 3.

Table 3. Reduced ODEs for nonlinear diffusion equations (7), $A_{u} \neq 0$.

\begin{tabular}{|l|l|c|c|l|}
\hline $\mathrm{N}$ & Subalgebra & Ansatz $u=$ & $\omega$ & \multicolumn{1}{|c|}{ Reduced ODE } \\
\hline 1 & $\left\langle Q_{1}\right\rangle$ & $\varphi(\omega)$ & $x$ & $\left(A(\varphi) \varphi^{\prime}\right)^{\prime}=0$ \\
2 & $\left\langle Q_{2}\right\rangle$ & $\varphi(\omega)$ & $t$ & $\varphi^{\prime}=0$ \\
3 & $\left\langle Q_{1}+\varepsilon Q_{2}\right\rangle$ & $\varphi(\omega)$ & $x-\varepsilon t$ & $\varepsilon \varphi^{\prime}=-\left(A(\varphi) \varphi^{\prime}\right)^{\prime}$ \\
4 & $\left\langle Q_{3}\right\rangle$ & $\varphi(\omega)$ & $x / \sqrt{t}$ & $\omega \varphi^{\prime}=-2\left(A(\varphi) \varphi^{\prime}\right)^{\prime}$ \\
\hline
\end{tabular}

The first three equations can be easily integrated for all values of $A(u)$. Solutions of the last equation are known for many functions $A(u)$ (see Section 9).

Consider now in more detail equations with wider symmetry algebras. Up to the group of equivalence transformations

$$
\tilde{t}=\varepsilon_{1} t+\varepsilon_{4}, \quad \tilde{x}=\varepsilon_{2} x+\varepsilon_{5}, \quad \tilde{u}=\varepsilon_{3} u+\varepsilon_{6}, \quad \tilde{A}=\varepsilon_{1}^{-1} \varepsilon_{2}^{2} A
$$

there exist three inequivalent cases of extensions of Lie symmetry algebra [44]: $a=e^{u}, a=u^{\mu}, \mu \neq-4 / 3$ and $a=u^{-4 / 3}$.

\section{Nonlinear diffusion equations. Exponential nonlinearity}

We start from the equation with exponential nonlinearity

$$
u_{t}=\left(e^{u} u_{x}\right)_{x}
$$

having the four-dimensional Lie algebra spanned by the operators

$$
Q_{1}=\partial_{t}, Q_{2}=t \partial_{t}-\partial_{u}, Q_{3}=\partial_{x}, Q_{4}=x \partial_{x}+2 \partial_{u} .
$$

The only non-zero commutators of these operators are $\left[Q_{1}, Q_{2}\right]=Q_{1}$ and $\left[Q_{3}, Q_{4}\right]=$ $Q_{3}$. Therefore $A^{\max }$ is a realization of the algebra $2 A_{2.1}[\mathbf{4 0}]$. All the possible inequivalent (with respect to inner automorphisms) one-dimensional subalgebras of $2 A_{2.1}$ [46] are exhausted by the ones listed in Table 4 .

Table 4. Reduced ODEs for (8). $\alpha \neq 0, \varepsilon= \pm 1, \delta=\operatorname{sign} t$.

\begin{tabular}{|l|l|c|c|l|}
\hline $\mathrm{N}$ & Subalgebra & Ansatz $u=$ & $\omega$ & \multicolumn{1}{|c|}{ Reduced ODE } \\
\hline 1 & $\left\langle Q_{3}\right\rangle$ & $\varphi(\omega)$ & $t$ & $\varphi^{\prime}=0$ \\
2 & $\left\langle Q_{4}\right\rangle$ & $\varphi(\omega)+2 \ln |x|$ & $t$ & $\varphi^{\prime}=2 e^{\varphi}$ \\
3 & $\left\langle Q_{1}\right\rangle$ & $\varphi(\omega)$ & $x$ & $\left(e^{\varphi}\right)^{\prime \prime}=0$ \\
4 & $\left\langle Q_{2}\right\rangle$ & $x$ & $\left(e^{\varphi}\right)^{\prime \prime}=-\delta$ \\
5 & $\left\langle Q_{1}+\varepsilon Q_{3}\right\rangle$ & $\varphi(\omega)-\ln |t|$ & $x-\varepsilon t$ & $\left(e^{\varphi}\right)^{\prime \prime}=-\varepsilon \varphi^{\prime}$ \\
6 & $\left\langle Q_{2}+\varepsilon Q_{3}\right\rangle$ & $\varphi(\omega)$ & $x-\varepsilon \ln |t|$ & $\left(e^{\varphi}\right)^{\prime \prime}=-\delta\left(\varepsilon \varphi^{\prime}+1\right)$ \\
7 & $\left\langle Q_{1}+\varepsilon Q_{4}\right\rangle$ & $\varphi(\omega)+2 \varepsilon t$ & $x e^{-\varepsilon t}$ & $\left(e^{\varphi}\right)^{\prime \prime}=-\varepsilon \omega \varphi^{\prime}+2 \varepsilon$ \\
8 & $\left\langle Q_{2}+\alpha Q_{4}\right\rangle$ & $\varphi(\omega)+(2 \alpha-1) \ln |t|$ & $x|t|-\alpha$ & $\left(e^{\varphi}\right)^{\prime \prime}=\delta\left(-\alpha \omega \varphi^{\prime}+2 \alpha-1\right)$ \\
\hline
\end{tabular}


Solving the equations $4.1-4.5$ we have the following solutions of (8):

$$
\begin{gathered}
u=\ln \left|c_{1} x+c_{0}\right|, \quad u=\ln \left(\frac{-x^{2}}{2 t}+\frac{c_{1} x+c_{0}}{t}\right), \\
u=\varphi(x-\varepsilon t) \quad \text { where } \int \frac{e^{\varphi}}{c_{1}-\varepsilon \varphi} d \varphi=x-\varepsilon t+c_{0} .
\end{gathered}
$$

Equation (8) admits an additive separation of variable that leads to the solution invariant with respect to scale transformation.

If $u=f(t, x)$ is a solution of equation (8), then

$$
\tilde{u}=f\left(\varepsilon_{1} t+\varepsilon_{3}, \varepsilon_{2} x+\varepsilon_{4}\right)-\varepsilon_{1}+2 \varepsilon_{2}
$$

is also solution of the same equation.

\section{Nonlinear diffusion equations. Power nonlinearities}

Another case of equations admitting extension of the Lie symmetry algebra is the one having power nonlinearity

$$
u_{t}=\left(|u|^{\mu} u_{x}\right)_{x}
$$

As in the previous cases the invariance algebra of (9)

$$
A^{\max }=\left\langle Q_{1}=\partial_{t}, Q_{2}=t \partial_{t}-\mu^{-1} u \partial_{u}, Q_{3}=\partial_{x}, Q_{4}=x \partial_{x}+2 \mu^{-1} u \partial_{u}\right\rangle
$$

is a realization of the algebra $2 A_{2.1}$.

If $u=f(t, x)$ is a solution of equation (9), then

$$
\tilde{u}=\varepsilon_{1}^{-1} \varepsilon_{2}^{2} f\left(\varepsilon_{1}^{\mu} t+\varepsilon_{3}, \varepsilon_{2}^{\mu} x+\varepsilon_{4}\right)
$$

is also solution of the same equation.

The result of reduction (9) under inequivalent subalgebras of $A^{\text {max }}$ is written down in Table 5.

Table 5. Reduced ODEs for (9). $\mu \neq 0 \alpha \neq 0, \varepsilon= \pm 1, \delta=\operatorname{sign} t$.

\begin{tabular}{|l|l|c|c|l|}
\hline $\mathrm{N}$ & Subalgebra & Ansatz $u=$ & $\omega$ & \multicolumn{1}{c|}{ Reduced ODE } \\
\hline 1 & $\left\langle Q_{3}\right\rangle$ & $\varphi(\omega)$ & $t$ & $\varphi^{\prime}=0$ \\
2 & $\left\langle Q_{4}\right\rangle$ & $\varphi(\omega)|x|^{2 / \mu}$ & $t$ & $\varphi^{\prime}=2 \mu^{-2}(2+\mu) \varphi^{\mu+1}$ \\
3 & $\left\langle Q_{1}\right\rangle$ & $\varphi(\omega)$ & $x$ & $\left(\varphi^{\mu} \varphi^{\prime}\right)^{\prime}=0$ \\
4 & $\left\langle Q_{2}\right\rangle$ & $\varphi(\omega)|t|^{-1 / \mu}$ & $x$ & $\left(\varphi^{\mu} \varphi^{\prime}\right)^{\prime}=-\delta \mu^{-1} \varphi$ \\
5 & $\left\langle Q_{1}+\varepsilon Q_{3}\right\rangle$ & $\varphi(\omega)$ & $x-\varepsilon t$ & $\left(\varphi^{\mu} \varphi^{\prime}\right)^{\prime}=-\varepsilon \varphi^{\prime}$ \\
6 & $\left\langle Q_{2}+\varepsilon Q_{3}\right\rangle$ & $\varphi(\omega)|t|^{-1 / \mu}$ & $x-\varepsilon \ln |t|$ & $\left(\varphi^{\mu} \varphi^{\prime}\right)^{\prime}=-\delta \varepsilon \varphi^{\prime}-\delta \mu^{-1} \varphi$ \\
7 & $\left\langle Q_{1}+\varepsilon Q_{4}\right\rangle$ & $\varphi(\omega) e^{2 \varepsilon \mu^{-1} t}$ & $x e^{-\varepsilon t}$ & $\left(\varphi^{\mu} \varphi^{\prime}\right)^{\prime}=-\varepsilon \omega \varphi^{\prime}+2 \mu^{-1} \varepsilon \varphi$ \\
8 & $\left\langle Q_{2}+\alpha Q_{4}\right\rangle$ & $\varphi(\omega)|t|^{(2 \alpha-1) / \mu}$ & $x|t|^{-\alpha}$ & $\left(\varphi^{\mu} \varphi^{\prime}\right)^{\prime}=\delta \mu^{-1}(2 \alpha-1) \varphi-\delta \alpha \omega \varphi^{\prime}$ \\
\hline
\end{tabular}


For some of the reduced equations the general solutions are known. For other ones we succeeded to find only particular solutions. These solutions are following:

$$
\begin{gathered}
u=\left|c_{1} x+c_{0}\right|^{\frac{1}{\mu+1}}, \quad u=\left(c_{0}-\varepsilon \mu(x-\varepsilon t)\right)^{\frac{1}{\mu}}, \\
u=\left(-\frac{\mu}{\mu+2} \frac{\left(x+c_{0}\right)^{2}}{2 t}+c_{1}|t|^{-\frac{\mu}{\mu+2}}\right)^{\frac{1}{\mu}}, \\
u=\left(-\frac{\mu}{\mu+2} \frac{\left(x+c_{0}\right)^{2}}{2 t}+c_{1}\left(x+c_{0}\right)^{\frac{\mu}{\mu+1}}|t|^{-\frac{\mu(2 \mu+3)}{2(\mu+1)^{2}}}\right)^{\frac{1}{\mu}}, \\
u=\varphi(x-\varepsilon t) \quad \text { where } \int \frac{\varphi^{\mu}}{c_{1}-\varepsilon \varphi} d \varphi=x-\varepsilon t+c_{0} .
\end{gathered}
$$

Equation (9) admits multiplicative separation of variables. Namely, for all values of $\mu$ one can find solution in form of the product of two functions of different arguments:

$$
u(t, x)=\left(b_{1} t+b_{0}\right)^{-1 / \mu} f(x),
$$

where the function $f=f(x)$ is given implicitly

$$
\int \frac{f^{\mu} d f}{\sqrt{c_{1}-\lambda f^{\mu+2}}}= \pm x+c_{0}, \quad \lambda=\frac{2 b_{1}}{\mu(\mu+2)} .
$$

These could be found also in $[\mathbf{2}, \mathbf{5}, \mathbf{6}, \mathbf{1 5}, \mathbf{2 4}, \mathbf{2 6}, \mathbf{4 8}, \mathbf{5 2}, \mathbf{5 8}, \mathbf{7 2}, \mathbf{7 4}]$. The most studied cases are equations with $\mu= \pm 1,-2,-4 / 3,-3 / 2$. Below we adduce their exact solutions that are inequivalent to (11) with respect to the Lie symmetry transformations.

Equation with the singular value of the parameter $\mu=-1$ called often the fast diffusion equation, is distinguished by the reduction procedure. Lie invariant solutions of it will be adduced in separate section together with non-Lie solutions obtained from invariance of the fast diffusion equation with respect to the nonclassical potential reduction operators.

Fujita-Storm equation (5) is linearizable and has been considered in a separate section.

Equation

$$
u_{t}=\left(u^{-4 / 3} u_{x}\right)_{x}
$$

admits the five-dimensional Lie symmetry algebra generated by

$Q_{1}=\partial_{t}, Q_{2}=t \partial_{t}-\mu^{-1} u \partial_{u}, Q_{3}=\partial_{x}, Q_{4}=x \partial_{x}+\frac{3}{2} u \partial_{u}, Q_{5}=-x^{2} \partial_{x}+3 x u \partial_{u}$

An optimal system of one-dimensional subalgebras of this algebra is

$$
\left\langle Q_{1}+Q_{4}\right\rangle,\left\langle a Q_{3}+Q_{4}\right\rangle,\left\langle Q_{5}\right\rangle,\left\langle Q_{2}+Q_{5}\right\rangle,\left\langle Q_{3}+Q_{5}\right\rangle .
$$

One can easily construct the corresponding ansatzes and the reduced ODEs. However, to the best of our knowledge all the found solutions of these equations are equivalent to (11) with particular value of parameter $\mu=-4 / 3$. Solutions of (13) invariant with respect to dilatation operators can be found also in [35]. Besides the already adduced Lie invariant solutions, equation (13) has functional separated solution $[\mathbf{2 2}, \mathbf{5 7}]$

$$
u=\left(\varphi_{4}(t) x^{4}+\varphi_{3}(t) x^{3}+\varphi_{2}(t) x^{2}+\varphi_{1}(t) x+\varphi_{0}(t)\right)^{-3 / 4},
$$


where the functions $\varphi_{i}=\varphi_{i}(t)$ are determined by the system of ordinary differential equations

$$
\begin{gathered}
\varphi_{0}^{\prime}=-\frac{3}{4} \varphi_{1}^{2}+2 \varphi_{0} \varphi_{2}, \quad \varphi_{1}^{\prime}=-\varphi_{1} \varphi_{2}+6 \varphi_{0} \varphi_{3}, \quad \varphi_{2}^{\prime}=-\varphi_{2}^{2}+\frac{3}{2} \varphi_{1} \varphi_{3}+12 \varphi_{0} \varphi_{4} \\
\varphi_{3}^{\prime}=-\varphi_{2} \varphi_{3}+6 \varphi_{1} \varphi_{4}, \quad \varphi_{4}^{\prime}=-\frac{3}{4} \varphi_{3}^{2}+2 \varphi_{2} \varphi_{4} .
\end{gathered}
$$

The general form of exact solutions of (13) obtained from the known ones $u=f(t, x)$ with action of group transformations is

$$
\tilde{u}=\frac{\varepsilon_{1}^{-1} \varepsilon_{2}^{2}}{\left(\varepsilon_{5} x+1\right)^{3}} f\left(\varepsilon_{1}^{-4 / 3} t+\varepsilon_{3}, \frac{\varepsilon_{2}^{-4 / 3} x}{\varepsilon_{5} x+1}+\varepsilon_{4}\right) .
$$

Equation

$$
u_{t}=\left(u^{-3 / 2} u_{x}\right)_{x}
$$

admits also the functional separation of variables. The corresponding exact solution is

$$
u=\left(3 c_{1} x^{3}+f_{2}(t) x^{2}+f_{1}(t) x+f_{0}(t)\right)^{-2 / 3} .
$$

Here

$$
\begin{aligned}
& f_{2}(t)=3 \int \varphi(t) d t+3 c_{2}, \quad f_{1}(t)=\frac{1}{c_{1}}\left(\int \varphi(t) d t+c_{2}\right)^{2}+\frac{1}{2 c_{1}} \varphi(t), \\
& f_{0}(t)=\frac{1}{9 c_{1}^{2}}\left(\int \varphi(t) d t+c_{2}\right)^{3}+\frac{1}{6 c_{1}^{2}} \varphi(t)\left(\int \varphi(t) d t+c_{2}\right)+\frac{1}{36 c_{1}^{2}} \varphi^{\prime}(t),
\end{aligned}
$$

where the function $\varphi=\varphi(t)$ is defined implicitly by $\int\left(c_{3}-8 \varphi^{3}\right)^{-1 / 2} d \varphi= \pm t+c_{4}$.

T.K. Amerov [3] and J.R. King [36] suggested to look for solutions of the equation

$$
u_{t}=\left(u^{-1 / 2} u_{x}\right)_{x}
$$

in the form $u=\left(\varphi^{1}(x) t+\varphi^{0}(x)\right)^{2}$ where the functions $\varphi^{1}(x)$ and $\varphi^{0}(x)$ satisfy the system of ODEs $\varphi_{x x}^{1}=\left(\varphi^{1}\right)^{2}, \varphi_{x x}^{0}=\varphi^{0} \varphi^{1}$. A particular solution of this system is

$$
\varphi^{1}=\frac{6}{x^{2}}, \quad \varphi^{0}=\frac{c_{1}}{x^{2}}+\frac{c_{2}}{x^{3}} .
$$

\section{Porous medium equation $u_{t}=\left(u u_{x}\right)_{x}$}

Another important subclass of diffusion equations is a special case of equation (9) with $\mu=1$

$$
u_{t}=\left(u u_{x}\right)_{x}
$$

called also porous medium equation. It first exact solution has been obtained by Boussinesq [13]. He was looking for a solution in a separated form $u(t, x)=$ $X(x) T(t)$ satisfying conditions

$$
u(t, 0)=0,\left.\quad u_{x}\right|_{x=L}=0 .
$$

Thus constructed solution reads as

$$
u=\frac{H_{0} F(\xi)}{1+\left(3 b^{2} H_{0} / 2 L^{2}\right) t},
$$

where $H_{0}$ is a constant, $\xi=x / L$ and the function $F=F(\xi)$ is defined implicitly

$$
\xi=\frac{1}{b} \int_{0}^{F} \frac{\lambda d \lambda}{\sqrt{1-\lambda^{3}}}, \quad b=\int_{0}^{1} \frac{\lambda d \lambda}{\sqrt{1-\lambda^{3}}}=\frac{1}{3} B\left(\frac{2}{3}, \frac{1}{2}\right) .
$$


The next exact solution of the porous medium equation (14) was found much later by Barenblatt [6] and written in the present form by Sokolov [59] (the instant source solution):

$$
u=\frac{1}{6 t}\left((9 t)^{2 / 3}-x^{2}\right), \quad 0 \leq x \leq(9 t)^{1 / 3}=l .
$$

It is easy to become convinced of the fact that both Boussinesq and Barenblatt solutions correspond to the Lie symmetry of the porous medium equation (14) [73]. Indeed, the Boussinesq solution is a particular case of the ansatz

$$
u=(1+\alpha t)^{-1} \phi(x), \quad \alpha=\frac{3 b^{2} H_{0}}{2 L^{2}},
$$

that is invariant with respect to the one-parameter Lie symmetry group generated by $Q=(1+\alpha t) \partial_{t}-u \partial_{u}$. Barenblatt solution is invariant with respect to the one-parameter Lie symmetry group generated by $Q=3 t \partial_{t}+x \partial_{x}-u \partial_{u}$.

Besides (11), its exact solutions in parametric form are known [48]:

$$
\begin{gathered}
x=\left(6 t+c_{1}\right) \xi+c_{2} \xi^{2}+c_{3}, \quad u=-\left(6 t+c_{1}\right) \xi^{2}-2 c_{2} \xi^{3}, \\
x=t f(\omega)+g(\omega), \quad u=t f^{\prime}(\omega)+g^{\prime}(\omega),
\end{gathered}
$$

where the functions $f=f(\omega)$ and $g=g(\omega)$ are determined by the system of ODEs:

$$
\left(f^{\prime}\right)^{2}-f f^{\prime \prime}=f^{\prime \prime \prime}, \quad f^{\prime} g^{\prime}-f g^{\prime \prime}=g^{\prime \prime \prime} .
$$

It is obvious, that the second equation has two linearly independent particular solutions $g=1$ and $g=f$. The general solution of these equations can be represented in form

$$
g=c_{1}+c_{2} f+c_{3}\left(f \int \psi d \omega-\int f \psi d \xi\right), \quad f=f(\omega), \quad \psi=\frac{1}{\left(f^{\prime}\right)^{2}} e^{-\int f d \omega} .
$$

It is not difficult to verify, that it has the following particular solutions

$$
f=\frac{6}{\omega+c_{1}} \text { and } f=c_{1} e^{c_{2} \omega} .
$$

One can see, that the first solution leads to the previously given implicit solution.

\section{Fast diffusion equation $u_{t}=\left(u^{-1} u_{x}\right)_{x}$}

All invariant solutions of fast diffusion equation

$$
\boldsymbol{u}_{\boldsymbol{t}}=\left(\boldsymbol{u}^{-1} \boldsymbol{u}_{\boldsymbol{x}}\right)_{\boldsymbol{x}},
$$

which were earlier constructed in closed forms with the classical Lie method, were collected e.g. in [48-51]. A complete list of $G_{1}$-inequivalent solutions of such type is exhausted by the following ones:
1) $u=\frac{1}{1+\varepsilon e^{x+t}}$,
2) $u=e^{x}$,
3) $u=\frac{1}{x-t+\mu t e^{-x / t}}$,
4) $u=\frac{2 t}{x^{2}+\varepsilon t^{2}}$,
5) $u=\frac{2 t}{\cos ^{2} x}$,
6) $u=\frac{-2 t}{\cosh ^{2} x}$,
7) $u=\frac{2 t}{\sinh ^{2} x}$.

The below arrows denote the possible transformations of solutions (16) to each other by means of the potential hodograph transformation (17) up to translations 
with respect to $x[\mathbf{5 0}]$ :

$$
\begin{array}{ll}
\circlearrowright 1)_{\varepsilon=0} ; & \left.\left.\left.\left.1)_{\varepsilon=1} \longleftrightarrow 1\right)_{\varepsilon=-1, x+t<0} ; \quad \circlearrowright 1\right)_{\varepsilon=-1, x+t>0} ; \quad 2\right) \longleftrightarrow 3\right)_{\mu=0, x>t} ; \\
\circlearrowright 4)_{\varepsilon=0} ; & \left.\left.\left.\left.5) \longleftrightarrow 4)_{\varepsilon=4} ; \quad 6\right) \longleftrightarrow 4\right)_{\varepsilon=-4,|x|<2|t|} ; \quad 7\right) \longleftrightarrow 4\right)_{\varepsilon=-4,|x|>2|t|} .
\end{array}
$$

The sixth connection can be found also in $[\mathbf{2 0 , 5 3}$. If $\mu \neq 0$ solution 3 ) from list (16) is mapped by (17) to the solution

$$
\text { 8) } u=t \vartheta(\omega)-t+\mu t e^{-\vartheta(\omega)}, \quad \omega=x-\ln |t| \text {, }
$$

which is invariant with respect to the algebra $\left\langle t \partial_{t}+\partial_{x}+u \partial_{u}\right\rangle$. Here $\vartheta$ is the function determined implicitly by the formula $\int\left(\vartheta-1+\mu e^{-\vartheta}\right)^{-1} d \vartheta=\omega$.

Some non-Lie exact solutions of $(15)$ were obtained in $[\mathbf{2 3}, \mathbf{5 5}, \mathbf{5 6}]$. Thus, P. Rosenau [56] found that potential equation $v_{t}=v_{x}{ }^{-1} v_{x x}$ corresponding to (15) admits, in addition to the usual variable separation $v=T(t) X(x)$, the additive one $v=Y(x+\lambda t)+Z(x-\lambda t)$ which is a potential additive variable separation for the fast diffusion equation (15). (The classical multiplicative separation of variables is given by (12) with $\mu=-1$.) To construct nonclassical solutions of (15), C. Qu [55] made use of generalized conditional symmetry method, looking for the conditional symmetry operators in the special form $Q=\left(u_{x x}+H(u) u_{x}{ }^{2}+F(u) u_{x}+G(u)\right) \partial_{u}$. M.L. Gandarias [23] investigated some families of usual and potential nonclassical symmetries of (9). In particular, using an ansatz for the coefficient $\eta$, she found non-trivial reduction operators in the so-called "no-go" case when the coefficient of $\partial_{t}$ vanishes, i.e. operators can be reduced to the form $Q=\partial_{x}+\eta(t, x, u) \partial_{u}$. These solutions and the ones similar to them were represented uniformly over the complex field as compositions of two simple waves which move with the same "velocities" in opposite directions in [51]. Using such representation the following solutions of fast diffusion equation (15) were obtained [51]:

$$
\begin{aligned}
& \left.1^{\prime}\right) u=\cot (x-t)-\cot (x+t)=\frac{2 \sin 2 t}{\cos 2 t-\cos 2 x}, \\
& \left.2^{\prime}\right) u=\operatorname{coth}(x-t)-\operatorname{coth}(x+t)=\frac{2 \sinh 2 t}{\cosh 2 x-\cosh 2 t}, \\
& \left.3^{\prime}\right) u=\operatorname{coth}(x-t)-\tanh (x+t)=\frac{2 \cosh 2 t}{\sinh 2 x-\sinh 2 t}, \\
& \left.4^{\prime}\right) u=\tanh (x-t)-\tanh (x+t)=-\frac{2 \sinh 2 t}{\cosh 2 x+\cosh 2 t}, \\
& \left.5^{\prime}\right) u=\cot (i x+t)-\cot (i x-t)=\frac{2 \sin 2 t}{\cosh 2 x-\cos 2 t}, \\
& \left.6^{\prime}\right) u=i \cot (x+i t)-i \cot (x-i t)=\frac{2 \sinh 2 t}{\cosh 2 t-\cos 2 x} .
\end{aligned}
$$

Transformation (17) acts on the set of solutions $\left.\left.1^{\prime}\right)-6^{\prime}\right)$ in the following way [51]:

$$
\begin{aligned}
& \left.\left.1^{\prime}\right)_{\cos 2 t<\cos 2 x} \longleftrightarrow 5^{\prime}\right)\left.\right|_{t \rightarrow t+\pi / 2, x \rightarrow x / 2, v \rightarrow 2 v} ; \\
& \left.\left.1^{\prime}\right)_{\cos 2 t>\cos 2 x} \longleftrightarrow 5^{\prime}\right)\left.\right|_{x \rightarrow x / 2, v \rightarrow 2 v-\pi} ; \\
& \left.\left.\left.2^{\prime}\right)_{|x|<|t|} \longleftrightarrow 4^{\prime}\right)\left.\right|_{x \rightarrow x / 2, v \rightarrow 2 v} ; \quad \circlearrowright 2^{\prime}\right)\left._{|x|>|t|}\right|_{x \rightarrow x / 2, v \rightarrow 2 v} ; \\
& \left.\left.\left.\circlearrowright 3^{\prime}\right)\left._{x<t}\right|_{x \rightarrow x / 2, v \rightarrow 2 v} ; \quad 3^{\prime}\right)_{x>t} \longleftrightarrow 3^{\prime}\right)\left._{x>t}\right|_{x \rightarrow-x / 2, v \rightarrow-2 v} ; \\
& \left.\circlearrowright 6^{\prime}\right)\left.\right|_{x \rightarrow x / 2, v \rightarrow 2 v} .
\end{aligned}
$$


These actions can be interpreted in terms of actions of transformation (17) on the nonclassical symmetry operators which correspond to solutions $\left.1^{\prime}\right)-6^{\prime}$ ).

In [56] P. Rosenau considered additive separation of variables for the potential fast diffusion equation (15) and constructed solution $4^{\prime}$ ). Using the generalized conditional symmetry method, C. Qu [55] found solutions which can be written in forms $1^{\prime}$ ) and $6^{\prime}$ ). After rectifying computations in two cases from [55], one can find also solutions $2^{\prime}$ ) and $5^{\prime}$ ). Solutions $1^{\prime}$ ), $3^{\prime}$ ) and $4^{\prime}$ ) were obtained in $[\mathbf{2 3}]$. The remaining solutions from the above list were found in [51].

One of techniques which can be applied for finding the above solutions is reduction by conditional symmetry operators of the form $Q=\partial_{x}+\left(\eta^{1}(t, x) u+\right.$ $\left.\eta^{2}(t, x)\right) u \partial_{u}$ (see $[\mathbf{2 3}]$ for details). All reductions performed with reduction operators of such type result in solutions which are equivalent to the listed Lie solutions 1)-7) or solutions $\left.\left.1^{\prime}\right)-6^{\prime}\right)$.

\section{Nonlinear diffusion equations. Other nonlinearities}

Known exact solutions of the reduced equation 3.4 corresponding to $\left\langle Q_{3}=\right.$ $\left.2 t \partial_{t}+x \partial_{x}\right\rangle$ (which are self-similar solutions of (7)) are adduced in Table $6[\mathbf{4 8}]$.

Table 6. Self-similar solutions for nonlinear diffusion equations $(7), \omega=x / \sqrt{t}$

\begin{tabular}{|l|c|c|l|}
\hline $\mathrm{N}$ & $A(u)$ & Solution $\omega=\omega(u)$ & Conditions \\
\hline 1 & $\frac{n}{2} u^{n}-\frac{n}{2(n+1)} u^{2 n}$ & $1-u^{n}$ & $n>0$ \\
\hline 2 & $\frac{n}{2(n+1)}\left((1-u)^{n-1}-(1-u)^{2 n}\right)$ & $(1-u)^{n}$ & $n>0$ \\
\hline 3 & $\frac{n}{2(1-n)} u^{-2 n}-\frac{n}{2} u^{-n}$ & $u^{-n}-1$ & $0<n<1$ \\
\hline 4 & $\frac{1}{2} \sin ^{2} \frac{\pi u}{2}$ & $\cos \frac{\pi u}{2}$ & \\
\hline 5 & $\frac{1}{8} \sin \pi u(\pi u+\sin \pi u)$ & $\cos ^{2} \frac{\pi u}{2}$ & \\
\hline 6 & $\frac{1}{16} \sin ^{2} \pi u(5+\cos \pi u)$ & $\cos ^{3} \frac{\pi u}{2}$ & \\
\hline 7 & $\frac{1}{2} \cos \frac{\pi u}{2}\left(\cos \frac{\pi u}{2}+\frac{\pi u}{2}-1\right)$ & $1-\sin \frac{\pi u}{2}$ & \\
\hline 8 & $\frac{u \arccos u+1}{2 \sqrt{1-u^{2}}}-\frac{1}{2}$ & $\arccos u$ & \\
\hline 9 & $\frac{\pi-2(1-u) \arcsin (1-u)}{4 \sqrt{2 u-u^{3}}}-\frac{1}{2}$ & $\arcsin (1-u)$ & \\
\hline 10 & $\frac{u \arcsin u}{4 \sqrt{1-u^{2}}+\frac{1}{4} u^{2}}$ & $\sqrt{1-u^{2}}$ & \\
\hline 11 & $\frac{1}{2}(1-\ln u)$ & $-\ln u$ & \\
\hline
\end{tabular}


Let us give some more examples of travelling wave solutions $[\mathbf{2 6}, \mathbf{4 8}, \mathbf{5 8}]$ :

$$
\begin{gathered}
\boldsymbol{u}_{\boldsymbol{t}}=\left(\sinh ^{2} \boldsymbol{u} \boldsymbol{u}_{\boldsymbol{x}}\right)_{\boldsymbol{x}}, \quad u=\operatorname{arccosh}^{2} \frac{ \pm x+c_{1}}{\sqrt{c_{2}-2 t}}, \\
\boldsymbol{u}_{\boldsymbol{t}}=\left(\cosh ^{\mathbf{2}} \boldsymbol{u} \boldsymbol{u}_{\boldsymbol{x}}\right)_{\boldsymbol{x}}, \quad u=\operatorname{arcsinh}^{2} \frac{ \pm x+c_{1}}{\sqrt{c_{2}-2 t}} \\
\boldsymbol{u}_{\boldsymbol{t}}=\left(\left(\boldsymbol{u}^{\mathbf{2} \boldsymbol{\mu}}+\boldsymbol{b} \boldsymbol{u}^{\boldsymbol{\mu}}\right) \boldsymbol{u}_{\boldsymbol{x}}\right)_{\boldsymbol{x}}, \quad u=\left(\sqrt{\frac{c_{1}}{a\left(c_{1}+1\right)\left(c_{2}-t\right)}}\left(x+c_{1}\right)-\frac{2 b c_{1}}{a\left(c_{1}+1\right)}\right)^{\frac{1}{\mu}} . \\
\boldsymbol{u}_{\boldsymbol{t}}=\left(\left(\boldsymbol{e}^{\mathbf{2 u}}+\boldsymbol{b u} \boldsymbol{u}^{\boldsymbol{u}}\right) \boldsymbol{u}_{\boldsymbol{x}}\right)_{\boldsymbol{x}}, \quad u=\ln \left(\frac{ \pm x+c_{1}}{\sqrt{c_{2}-2 t}}-b\right) . \\
\boldsymbol{u}_{\boldsymbol{t}}=\left(\boldsymbol{u} \boldsymbol{e}^{\boldsymbol{u}} \boldsymbol{u}_{\boldsymbol{x}}\right)_{\boldsymbol{x}}, \quad u=\ln \left(c_{1} x+c_{1}^{2} t+c_{0}\right) .
\end{gathered}
$$

For equation with logarithmical nonlinearity

$$
u_{t}=\left(\ln u u_{x}\right)_{x}
$$

travelling wave and self-similar solutions are known:

$$
u=\exp \left( \pm \sqrt{2 c_{1} x+2 c_{1}^{2} t+c_{2}}\right), \quad u=\exp \left(\frac{ \pm x+c_{1}}{c_{2}-2 t}-1\right) .
$$

A number of exact solution for equations of class (7) were constructed with nonlocal (quasilocal or potential) symmetries $[\mathbf{2}, \mathbf{1 1}, \mathbf{1 2}, \mathbf{6 0}-\mathbf{6 2}]$.

Thus, e.g., reductions with respect to the optimal system of subalgebras of Lie algebra of potential/quasilocal symmetries of equation

$$
\left.u_{t}=\left(\left(1+u^{2}\right)^{-1} u_{x}\right)\right)_{x}
$$

give rise to exact solutions of form

$$
\begin{gathered}
u=c e^{t-x}\left(1-c^{2} e^{2(t-x)}\right)^{-1 / 2}, \quad u=-x\left(c-2 t-x^{2}\right)^{-1 / 2}, \\
u=\tan (\varphi(\omega)+\arctan (\lambda(\omega))+\varepsilon t), \\
\text { where } \quad \begin{aligned}
\omega & =x^{2}+v^{2}, \quad v=\tan (\varphi(\omega)+\varepsilon t), \quad \text { and } \\
u & =x \tan (\varphi(\omega)+\arctan (\lambda(\omega))+\varepsilon t), \\
\text { where } \quad \omega & =\frac{x^{2}+v^{2}}{t}, \quad v=x \tan \left(\varphi(\omega)+\frac{\alpha}{2} \varepsilon t\right) .
\end{aligned}
\end{gathered}
$$

Here $\varphi(\omega)$ and $\lambda(\omega)$ are arbitrary solutions of the system $\varphi^{\prime}=\omega^{-1} \lambda / 2, \lambda^{\prime}=$ $\left(1+\lambda^{2}\right)\left(\varepsilon-\omega^{-1} \lambda\right) / 2$. The list of known exact solutions of this equation (called often the Fujita's equation) involves also the following ones $[\mathbf{2}, \mathbf{1 6}, \mathbf{2 6}, \mathbf{4 8}]$ :

$$
\begin{aligned}
& u=\tan \left(c_{1} x+c_{2}\right), \quad u= \pm x\left(c_{1}-2 t-x^{2}\right)^{-1 / 2}, \\
& u=\frac{ \pm e^{t-x}}{\sqrt{1-e^{2(t-x)}}}, \quad \varepsilon(x+\varepsilon t)+c_{2}=\frac{1}{c_{1}^{2}+1}\left(\ln \frac{\left|u+c_{1}\right|}{\sqrt{u^{2}+1}}+c_{1} \arctan u\right), \\
& u=\frac{v}{\sqrt{1-v^{2}}}, \quad \text { where } \quad v=\frac{c_{1} e^{\lambda x}+c_{2} e^{-\lambda x}}{\sqrt{4 c_{1} c_{2}+c_{3} e^{-2 \lambda^{2} t}}} \text { or } \\
& v=\frac{c_{1} \sin \lambda x+c_{2} \cos \lambda x}{\sqrt{c_{1}^{2}+c_{2}^{2}+c_{3} e^{2 \lambda^{2} t}}} \\
& u=\frac{\sinh x}{\sqrt{-\cosh ^{2} x-e^{-2 t}}}, \quad u=\frac{ \pm \cosh x}{\sqrt{-\sinh ^{2} x+e^{-2 t}}}, \quad u=\frac{\sin x}{\sqrt{\cos ^{2} x \pm e^{2 t}}} .
\end{aligned}
$$


We adduce also some exact solutions of another equation with Fujita's type nonlinearity

$$
\left.u_{t}=\left(\left(1-u^{2}\right)^{-1} u_{x}\right)\right)_{x},
$$

namely $[\mathbf{1 6}]$ :

$$
\begin{gathered}
u=c, \quad u=\tanh x, \quad u=\frac{x}{\sqrt{x^{2}+2 t}}(t>0), \\
u=\frac{ \pm e^{t-x}}{\sqrt{1+e^{2(x-t)}}}, \quad u=\frac{\sinh x}{\sqrt{\cosh ^{2} x+e^{-2 t}}}, \\
u=\frac{\sinh x}{\sqrt{\cosh ^{2} x-e^{-2 t}}}(t>0), \quad u=\frac{ \pm \cosh x}{\sqrt{\sinh ^{2} x+e^{-2 t}}}(t<0), \\
u=\frac{\sin x}{\sqrt{-\cos ^{2} x+e^{2 t}}}
\end{gathered}
$$

The third similarity solution converges (pointwise) to a step function as $t \rightarrow 0^{+}$, and to zero as $t \rightarrow \infty$. The fourth solutions are bounded travelling waves. The fifth and sixth solutions converge to the time independent second solution as $t \rightarrow \infty$. The sixth solution converge to a step function as $t \rightarrow 0^{+}$. The seventh solution converges to the values \pm 1 as $t \rightarrow 0-$. The last solution converges to a square wave as $t \rightarrow 0+$, and to zero as $t \rightarrow \infty$.

One more example of solution obtained with application of potential symmetry is $u=\tan \left(\varphi(\omega)+\arctan \left(2 \omega \varphi^{\prime}\right)-\lambda^{-1} \ln t\right)$ for the equation

$$
\left.u_{t}=\left(\left(1+u^{2}\right)^{-1} e^{\lambda \arctan u} u_{x}\right)\right)_{x} .
$$

Here

$$
\omega=x^{2}+v^{2}, \quad \varphi=\frac{1}{\lambda} \ln \frac{c-\omega}{2}-\arctan \psi(\omega), \quad \frac{\psi^{\prime}}{1+\psi^{2}}+\frac{\psi}{2 \omega}+\frac{2}{\lambda(c-\omega)}=0 .
$$

All the potential symmetries of equations from class (18) can be obtained from Lie symmetries of (18) by means of prolongation to the potential variable $v$ and application of potential equivalence transformations [50]

$$
\tilde{t}=t, \quad \tilde{x}=x+\varepsilon v, \quad \tilde{u}=\frac{u}{1+\varepsilon u}, \quad \tilde{v}=v, \quad \tilde{A}=(1+\varepsilon u)^{2} A
$$

and hodograph transformation

$$
\tilde{t}=t, \quad \tilde{x}=v, \quad \tilde{u}=u^{-1}, \quad \tilde{v}=x, \quad \tilde{A}=u^{2} A,
$$

where $v_{x}=u, v_{t}=A u_{x}$.

Therefore, these transformations can be used for obtaining potentially invariant exact solutions from the Lie ones. The complete list of nonlinear constant coefficient diffusion and diffusion-convection equations having potential symmetries together with the transformations mapping them to the equations with power and exponential nonlinearities can be found in [50].

In [65] non-point nonclassical symmetry operators are used to obtain exact solutions of evolution equations. In particular, it is shown that equation

$$
\left.u_{t}=\left(u^{-2} e^{1 / u} u_{x}\right)\right)_{x}
$$


admits an exact solution in implicit form $u=\left(z^{2}+c\right) /(2 t)$, where

$$
\begin{gathered}
z=\frac{z}{\ln \frac{z^{2}+c}{2 e^{2} t}+\frac{2 \sqrt{c}}{z} \arctan \frac{z}{\sqrt{c}}+\frac{c_{1}}{z}}, \quad \text { if } \quad c>0, \quad \text { and } \\
z=\frac{z}{\ln \frac{z^{2}+c}{2 e^{2} t}+\frac{2 \sqrt{-c}}{z} \ln \frac{z-\sqrt{-c}}{z+\sqrt{-c}}+\frac{c_{1}}{z}}, \quad \text { if } \quad c<0 .
\end{gathered}
$$

A number of authors considered additive separated solutions of diffusion equations (7), i.e., solutions of form

$$
u(t, x)=\varphi(t)+\psi(x) .
$$

Usually such solutions are Lie invariant. They were adduced in previous sections. So, here we adduce only list of equations admitting such kind of separation of variables. Namely, a diffusion equation (7) admits separation of variables if and only if it is $G^{\sim}$-equivalent to equation with the diffusion coefficient being of the following functions $[\mathbf{1 6}]$ :

$$
\begin{gathered}
A=|u|^{\mu}, \quad A=e^{u}, \quad A=\left(u^{2} \pm 1\right)^{-1}, \\
A=z(u) e^{z(u)}, \quad u=\int_{1}^{z} s^{-3 / 2} e^{-s / 2} d s, \quad z>0, \\
A=e^{\sigma z(u)} \cosh z(u), \quad \sigma \neq \pm 1, \quad u=\int_{0}^{z} \cosh ^{-3 / 2} s e^{-\sigma s / 2} d s, \quad-\infty<z<\infty \\
A=e^{\sigma z(u)} \sinh z(u), \quad \sigma \neq \pm 1, \quad u=\int_{1}^{z} \sinh ^{-3 / 2} s e^{-\sigma s / 2} d s, \quad z>0, \\
A=e^{\sigma z(u)} \cos z(u), \quad u=\int_{0}^{z} \cos ^{-3 / 2} \operatorname{se}^{-\sigma s / 2} d s, \quad-\pi / 2<z<\pi / 2 .
\end{gathered}
$$

\section{Constant coefficient diffusion-convection equations}

Lie symmetries of the constant coefficient diffusion-convection equation

$$
u_{t}=\left(A(u) u_{x}\right)_{x}+B(u) u_{x}
$$

$B \neq 0$ and corresponding Lie reductions were considered by a number of authors, see for example, $[\mathbf{1 7}, \mathbf{3 2}, \mathbf{3 3}, \mathbf{4 3}, \mathbf{7 1}]$. However the complete group classification of class (18) was presented only recently in [49].

Any equivalence transformation of class (18) has the form:

$$
\begin{aligned}
& \tilde{t}=t \varepsilon_{4}^{2} \varepsilon_{5}+\varepsilon_{1}, \quad \tilde{x}=x \varepsilon_{4}+\varepsilon_{7} t+\varepsilon_{2}, \quad \tilde{u}=u \varepsilon_{6}+\varepsilon_{3}, \\
& \tilde{A}=A \varepsilon_{5}^{-1}, \quad \tilde{B}=B \varepsilon_{4}^{-1} \varepsilon_{5}^{-1}-\varepsilon_{7},
\end{aligned}
$$

where $\varepsilon_{1}, \ldots, \varepsilon_{7}$ are arbitrary constants, $\varepsilon_{4} \varepsilon_{5} \varepsilon_{6} \neq 0$. Note, that all the equations with $B(u)=$ const are reducible to diffusion equation (7). Besides such 'trivial' cases, Burgers equation and Fokas-Yortsos equation, only few Lie invariant solutions for equations with non-zero convectivity are found. Thus, e.g., in [71] scale-invariant solution

$$
x u^{-\mu / 2}-(1+2 / \mu) \ln \left(x u^{-\mu / 2}-1-2 / \mu\right)=c_{1} t+c_{0}
$$

in implicit form is found for the equation with power nonlinearities

$$
u_{t}=\left(u^{\mu} u_{x}\right)_{x}+u^{\mu / 2} u_{x}
$$

was found. 
M. Edwards [17] investigated Lie symmetries of (18) and constructed optimal subalgebras of the symmetry algebras for some of equations from the class. Here we supplement her results and adduce the complete list of Lie reductions of equations from class (18). (The linearizable Fokas-Yortsos and Burgers equations have been considered separately in Section 3.)

Table 7. Reduced ODEs for $u_{t}=\left(e^{\mu u} u_{x}\right)_{x}+e^{u} u_{x}(\mu \neq 0)$

\begin{tabular}{|l|c|c|l|}
\hline Subalgebra & Ansatz $u=$ & $\omega$ & \multicolumn{1}{c|}{ Reduced ODE } \\
\hline$\left\langle\partial_{x}\right\rangle$ & $\varphi(\omega)$ & $t$ & $\varphi^{\prime}=0$ \\
$\left\langle\partial_{t}\right\rangle$ & $\varphi(\omega)$ & $x$ & $\left(e^{\mu \varphi} \varphi^{\prime}\right)^{\prime}+e^{\varphi} \varphi^{\prime}=0$ \\
$\left\langle\partial_{t}+\varepsilon \partial_{x}\right\rangle$ & $\varphi(\omega)$ & $x-\varepsilon t$ & $-\varepsilon \varphi^{\prime}=\left(e^{\mu \varphi} \varphi^{\prime}\right)^{\prime}+e^{\varphi} \varphi^{\prime}$ \\
$\left\langle(\mu t-2 t+\varepsilon) \partial_{t}\right.$ & $\varphi(\omega)+$ & $x((\mu-2) t$ & $\varphi^{\prime}(1-\mu) \omega+1$ \\
$\left.+(\mu-1) x \partial_{x}+\partial_{u}\right\rangle(\mu \neq 2)$ & $+\frac{\ln ((\mu-2) t+\varepsilon)}{\mu-2}$ & $+\varepsilon)^{\frac{1-\mu}{\mu-2}}$ & $=\left(e^{\mu \varphi} \varphi^{\prime}\right)^{\prime}+e^{\varphi} \varphi^{\prime}$ \\
$\left\langle\varepsilon \partial_{t}+x \partial_{x}+\partial_{u}\right\rangle(\mu=2)$ & $\varphi(\omega)+\frac{t}{\varepsilon}$ & $x e^{-t / \varepsilon}$ & $\frac{1}{\varepsilon}-\varphi^{\prime} \omega=\left(e^{2 \varphi} \varphi^{\prime}\right)^{\prime}+e^{\varphi} \varphi^{\prime}$ \\
\hline
\end{tabular}

If $u=f(t, x)$ is an exact solution of equation $u_{t}=\left(e^{\mu u} u_{x}\right)_{x}+e^{u} u_{x}$, then

$$
u=f\left(e^{(\mu-2) \varepsilon_{1}} t+\varepsilon_{3}, e^{(\mu-1) \varepsilon_{1}+\varepsilon_{2}} x+\varepsilon_{4}\right)+\varepsilon_{1}+\varepsilon_{2}
$$

is also solution of the same equation.

Table 8. Reduced ODEs for $u_{t}=\left(e^{u} u_{x}\right)_{x}+u u_{x}$.

\begin{tabular}{|l|c|c|l|}
\hline Subalgebra & Ansatz $u=$ & $\omega$ & \multicolumn{1}{c|}{ Reduced ODE } \\
\hline$\left\langle\partial_{x}\right\rangle$ & $\varphi(\omega)$ & $t$ & $\varphi^{\prime}=0$ \\
$\left\langle\partial_{t}\right\rangle$ & $\varphi(\omega)$ & $x$ & $\left(e^{\varphi} \varphi^{\prime}\right)^{\prime}+\varphi \varphi^{\prime}=0$ \\
$\left\langle\partial_{t}+\varepsilon \partial_{x}\right\rangle$ & $\varphi(\omega)$ & $x-\varepsilon t$ & $-\varepsilon \varphi^{\prime}=\left(e^{\varphi} \varphi^{\prime}\right)^{\prime}+\varphi \varphi^{\prime}$ \\
$\left\langle(t+\varepsilon) \partial_{t}+(x-t) \partial_{x}+\partial_{u}\right\rangle$ & $\varphi(\omega)$ & $\frac{x+\varepsilon}{t+\varepsilon}$ & $1+\varphi^{\prime}(1-\omega)=\left(e^{\varphi} \varphi^{\prime}\right)^{\prime}+\varphi \varphi^{\prime}$ \\
& $+\ln |t+\varepsilon|$ & $+\ln |t+\varepsilon|$ & \\
\hline
\end{tabular}

If $u=f(t, x)$ is an exact solution of equation $u_{t}=\left(e^{u} u_{x}\right)_{x}+u u_{x}$, then

$$
u=f\left(e^{\varepsilon_{1}} t+\varepsilon_{2}, e^{\varepsilon_{1}}\left(x-\varepsilon_{1} t+\varepsilon_{3}\right)\right)+\varepsilon_{1}
$$

is also solution of the same equation.

Table 9. Reduced ODEs for $u_{t}=\left(u^{\mu} u_{x}\right)_{x}+u^{\nu} u_{x}$.

\begin{tabular}{|l|c|c|l|}
\hline Subalgebra & Ansatz $u=$ & $\omega$ & \multicolumn{1}{c|}{ Reduced ODE } \\
\hline$\left\langle\partial_{x}\right\rangle$ & $\varphi(\omega)$ & $t$ & $\varphi^{\prime}=0$ \\
$\left\langle\partial_{t}\right\rangle$ & $\varphi(\omega)$ & $x$ & $\left(\varphi^{\mu} \varphi\right)^{\prime}+\varphi^{\nu} \varphi^{\prime}=0$ \\
$\left\langle\partial_{t}+\varepsilon \partial_{x}\right\rangle$ & $\varphi(\omega)$ & $x-\varepsilon t$ & $-\varepsilon \varphi^{\prime}=\left(\varphi^{\mu} \varphi^{\prime}\right)^{\prime}+\varphi^{\nu} \varphi^{\prime}$ \\
$\left\langle(\mu t-2 \nu t+\varepsilon) \partial_{t}\right.$ & $\varphi(\omega)((\mu-2 \nu) t$ & $x((\mu-2 \nu) t$ & $\varphi+\varphi^{\prime} \omega=\left(\varphi^{\mu} \varphi^{\prime}\right)^{\prime}+\varphi^{\nu} \varphi^{\prime}$ \\
$\left.+(\mu-\nu) x \partial_{x}+u \partial_{u}\right\rangle,(\mu \neq 2 \nu)$ & $+\varepsilon)^{\frac{1}{\mu-2 \nu}}$ & $+\varepsilon)^{\frac{\nu-\mu}{\mu-2 \nu}}$ & \\
$\left\langle\varepsilon \partial_{t}+\nu x \partial_{x}+u \partial_{u}\right\rangle,(\mu=2 \nu)$ & $\varphi(\omega) e^{t / \varepsilon}$ & $x e^{-\nu t / \varepsilon}$ & $\varphi-\varphi^{\prime} \omega=\left(\varphi^{2 \nu} \varphi^{\prime}\right)^{\prime}+\varphi^{\nu} \varphi^{\prime}$ \\
\hline
\end{tabular}

If $u=f(t, x)$ is an exact solution of equation $u_{t}=\left(u^{\mu} u_{x}\right)_{x}+u^{\nu} u_{x}$, then

$$
u=e^{\varepsilon_{1}+\varepsilon_{2}} f\left(e^{(\mu-2 \nu) \varepsilon_{1}} t+\varepsilon_{3}, e^{(\mu-\nu) \varepsilon_{1}+\nu \varepsilon_{2}} x+\varepsilon_{4}\right)
$$

is also solution of the same equation. 
For equations with $\nu=\mu$ the generalized travelling wave solution is known:

$$
u=\left(\frac{c_{2}-x}{t+c_{1}}+\frac{\ln \left|t+c_{1}\right|}{\mu\left(t+c_{1}\right)}\right)^{1 / \mu} .
$$

If $\mu=2, \nu=1$ then the generalized travelling wave solution in implicit form is the following:

$$
2 \int \frac{u^{2} d u}{-u^{2}-2 \varepsilon u+c_{1}}=x-\varepsilon t+c_{2} .
$$

For such values $\mu$ and $\nu$ the degenerate solution linear in $x$ has the form $u=$ $\tau(t)\left(x+c_{1}\right)$, where function $\tau$ is given in implicit form

$$
-\frac{1}{\tau}+2 \ln \left|\frac{2 \tau+1}{\tau}\right|=t+c_{2}
$$

Table 10. Reduced ODEs for $u_{t}=\left(u^{\mu} u_{x}\right)_{x}+\ln u u_{x}$.

\begin{tabular}{|l|c|c|l|}
\hline Subalgebra & Ansatz $u=$ & $\omega$ & \multicolumn{1}{c|}{ Reduced ODE } \\
\hline$\left\langle\partial_{x}\right\rangle$ & $\varphi(\omega)$ & $t$ & $\varphi^{\prime}=0$ \\
$\left\langle\partial_{t}\right\rangle$ & $\varphi(\omega)$ & $x$ & $\left(\varphi^{\mu} \varphi\right)^{\prime}+\ln \varphi \varphi^{\prime}=0$ \\
$\left\langle\partial_{x}+\varepsilon \partial_{t}\right\rangle$ & $\varphi(\omega)$ & $x-\varepsilon t$ & $-\varepsilon \varphi^{\prime}=\left(\varphi^{\mu} \varphi\right)^{\prime}+\ln \varphi \varphi^{\prime}$ \\
$\left\langle(\mu t+\varepsilon) \partial_{t}\right.$ & $\varphi(\omega)(\mu t+\varepsilon)^{1 / \mu}$ & $\frac{\mu^{2} x+\varepsilon}{\mu^{2}(\mu t+\varepsilon)}+\frac{\ln |\mu t+\varepsilon|}{\mu^{2}}$ & $\frac{1}{\mu} \varphi-\mu \omega \varphi^{\prime}$ \\
$\left.+(\mu x-t) \partial_{x}+u \partial_{u}\right\rangle$ & & & $=\left(\varphi^{\mu} \varphi\right)^{\prime}+\ln \varphi \varphi^{\prime}$ \\
\hline
\end{tabular}

If $u=f(t, x)$ is an exact solution of equation $\boldsymbol{u}_{\boldsymbol{t}}=\left(\boldsymbol{u}^{\boldsymbol{\mu}} \boldsymbol{u}_{\boldsymbol{x}}\right)_{\boldsymbol{x}}+\ln \boldsymbol{u} \boldsymbol{u}_{\boldsymbol{x}}$, then

$$
u=e^{\varepsilon_{1}} f\left(e^{\mu \varepsilon_{1}} t+\varepsilon_{2}, e^{\mu \varepsilon_{1}}\left(x-\varepsilon_{1} t+\varepsilon_{3}\right)\right)
$$

is also solution of the same equation.

In particular, if $\mu=0$, we obtain $\boldsymbol{u}_{\boldsymbol{t}}=\boldsymbol{u}_{\boldsymbol{x} \boldsymbol{x}}+\ln \boldsymbol{u} \boldsymbol{u}_{\boldsymbol{x}}$. This equation has two known travelling wave solutions (usual and generalized ones):

$$
u(t, x)=\exp \left(c_{1} e^{-x+c_{2} t}+1-c_{2}\right), \quad u(t, x)=\exp \left(\frac{c_{1}-x}{t+c_{2}}+\frac{\ln \left|t+c_{2}\right|}{t+c_{2}}\right) .
$$

Generalized travelling wave solution are known for the following equations:

$$
\begin{gathered}
\boldsymbol{u}_{\boldsymbol{t}}=\left(\left(\boldsymbol{u}^{\mathbf{2 \mu}}+\boldsymbol{b} \boldsymbol{u}^{\boldsymbol{\mu}}\right) \boldsymbol{u}_{\boldsymbol{x}}\right)_{\boldsymbol{x}}+\boldsymbol{u}^{\boldsymbol{\mu}} \boldsymbol{u}_{\boldsymbol{x}}, \quad u=\left(x \varphi(t)+c_{1} \varphi(t)+\frac{b}{n} \varphi(t) \int \varphi(t) d t\right)^{1 / \mu} . \\
\boldsymbol{u}_{\boldsymbol{t}}=\left(\left(\boldsymbol{a} \boldsymbol{e}^{\mathbf{2 u}}+\boldsymbol{b} \boldsymbol{e}^{\boldsymbol{u}}\right) \boldsymbol{u}_{\boldsymbol{x}}\right)_{\boldsymbol{x}}+\boldsymbol{e}^{\boldsymbol{u}_{\boldsymbol{x}},}, u=\ln \left(x \varphi(t)+c_{1} \varphi(t)+b \varphi(t) \int \varphi(t) d t\right),
\end{gathered}
$$

where $\varphi=\varphi(t)$ is determined by the equation $\varphi^{\prime}=a \varphi^{3}+\varphi^{2}$.

\section{1. $n$-dimensional radially symmetric nonlinear diffusion equations}

Class (1) contains a subclass of physically important $n$-dimensional radially symmetric nonlinear diffusion equations. Preserving the common terminology we use for them the notation

$$
u_{t}=r^{1-n}\left(r^{n-1} A(u) u_{r}\right)_{r} .
$$

Equations of the form (20), especially for power nonlinearity

$$
u_{t}=r^{1-n}\left(r^{n-1} u^{\mu} u_{r}\right)_{r}
$$

have a large number of applications, for both $\mu>0$ (slow diffusion) and $\mu<0$ (fast diffusion). 
To the best of our knowledge, first similar solutions of (20) were instantaneous source-type solutions $[\mathbf{6}, \mathbf{4 7}]$ namely, solutions of form

$$
u=t^{-n /(\mu n+2)} f(\xi), \quad \xi=r t^{-1 /(\mu n+2)}
$$

if $\mu \neq-2 / N$ and

$$
u=e^{-\lambda n t} f(\xi), \quad \xi=r e^{\lambda t},
$$

where $\lambda$ is an arbitrary constant, in case $\mu=-2 / N$. Substituting these values into (21) yields (assuming $t>0$ in the first three cases):

$$
\begin{array}{lll}
\mu>0: & u=\left\{\begin{array}{ll}
t^{-\frac{n}{\mu n+2}}\left[\frac{\mu}{2(\mu n+2)}\left(a^{2}-r^{2} t^{-\frac{2}{\mu n+2}}\right)\right]^{\frac{1}{\mu}}, & r<a t^{\frac{1}{\mu n+2}} \\
0, & r \geq a t^{\frac{1}{\mu n+2}}
\end{array},\right. \\
\mu=0: & u=A t^{-n / 2} e^{-r^{2} / 4 t}, \\
0>\mu>-\frac{2}{n}: & u=t^{-\frac{n}{\mu n+2}\left[\frac{-\mu}{2(\mu n+2)}\left(a^{2}+r^{2} t^{-2 /(\mu n+2)}\right)\right]^{\frac{1}{\mu}},} \\
\mu=-\frac{2}{n}: & u=e^{-\lambda n t}\left[\frac{\lambda}{n}\left(a^{2}+r^{2} e^{-2 \lambda t}\right)\right]^{-n / 2}, \\
\mu<-\frac{2}{n}: & u=\left\{\begin{array}{ll}
(-t)^{-\frac{n}{\mu n+2}}\left[\frac{\mu}{2(\mu n+2)}\left(a^{2}+r^{2}(-t)^{-\frac{2}{\mu n+2}}\right)\right]^{\frac{1}{\mu}}, & t<0 \\
0, & t \geq 0
\end{array} .\right.
\end{array}
$$

The following instantaneous source-type solutions for equations (21) were obtained by King [34]:

Case $\mu=-1, n \neq-2: \quad f=(\mu n+2) \exp \left(-\frac{\alpha \xi^{-2-n}}{2-n}\right) / \int \xi \exp \left(-\frac{\alpha \xi^{-2-n}}{2-n}\right) d \xi$.

Case $\mu=-1, n=1: \quad f=1 /\left(\beta e^{-\alpha \xi}-\alpha^{-2}(\mu n+2)^{-1}(1+\alpha \xi)\right)$.

Case $\mu=-1, n=2: \quad f=1 /\left(\beta \xi^{\alpha}+\xi^{2}(\mu n+2)^{-1}(2-\alpha)^{-1}\right)$, if $\alpha \neq 2$ and

$$
f=1 /\left(\beta \xi^{2}+\xi^{2}(\mu n+2)^{-1} \ln \xi \text {, if } \alpha=2\right. \text {. }
$$

Case $\mu=-1, n=3: \quad f=2 /\left(2 \beta e^{-\alpha / \xi}+\left(\xi(\alpha+\xi)+\alpha^{-2} e^{-\alpha / \xi} E_{1}(-\alpha / \xi)\right)(\mu n+\right.$ $2)^{-1}$ ), where $E_{1}(z)=\int_{z}^{\infty} e^{-t} t^{-1} d t$ is the exponential integral.

Case $\mu=-2 / n: \quad f=\xi^{-n} g$, where $\int\left(n g-\alpha g^{2 / n}-\lambda g^{1+2 / n}\right) d g=\ln \xi$.

Case $\mu=-1 / 2$ :

$$
\begin{gathered}
f=\alpha(\mu n+2) \xi^{-n}\left(\beta J_{\nu-1}\left(\frac{\alpha^{1 / 2} \xi^{(4-n) / 2}}{(\mu n+2)^{1 / 2}(4-n)}\right)+\right. \\
\left.+(1-\beta) Y_{\nu-1}\left(\frac{\alpha^{1 / 2} \xi^{(4-n) / 2}}{(\mu n+2)^{1 / 2}(4-n)}\right)\right)^{2} \\
\times\left(\beta J_{\nu}\left(\frac{\alpha^{1 / 2} \xi^{(4-n) / 2}}{(\mu n+2)^{1 / 2}(4-n)}\right)+(1-\beta) Y_{\nu}\left(\frac{\alpha^{1 / 2} \xi^{(4-n) / 2}}{(\mu n+2)^{1 / 2}(4-n)}\right)\right)^{-2},
\end{gathered}
$$

where $\nu=2 /(4-n), J_{\nu}(z)$ and $Y_{\nu}(z)$ are Bessel functions of the first and the second type correspondingly.

We now consider solutions [34] that generalize the one-dimensional dipole solutions given for $\mu>0$ in [7]. The similarity variables are chosen to fix $\int_{o}^{\infty} r u d r$ 
in time (if the integral exists). In one dimension this corresponds to the centre of mass. The appropriate similarity solution to (21) then takes the form

$$
\begin{gathered}
u=t^{-1 /(\mu+1)} f(\xi), \quad \xi=r t^{-1 /(2(\mu+1))}, \quad n \neq-1, \\
u=e^{-2 \lambda t} f(\xi), \quad \xi=r e^{-\lambda t}, \quad n=-1 .
\end{gathered}
$$

For different values $n$ and $\mu$ the following solutions are known $[\mathbf{7}, \mathbf{3 4}]$ :

Case $\mu \neq 0,-2 / n: \quad f=\xi^{(2-n) /(\mu+1)}\left(\frac{n}{2(\mu n+2)}\left(\beta-\xi^{(\mu n+2) /(\mu+1)}\right)\right)^{1 / n}$.

Case $\mu=0: \quad f=\beta \xi^{2-n} e^{-\xi^{2} / 4}$.

Case $\mu=-2 / n: \quad f=\left(\frac{\xi^{2}}{n-2} \ln \frac{\xi}{\beta}\right)^{-n / 2}$.

Case $\mu=-1 / 2$ : (here $\nu=2-n / 2)$

$$
\begin{aligned}
f= & \frac{\alpha}{\xi^{2}}\left[\beta J_{\nu-1}\left(\frac{\alpha^{1 / 2} \xi}{2}\right)+(1-\beta) Y_{\nu-1}\left(\frac{\alpha^{1 / 2} \xi}{2}\right)\right]^{2} \times \\
& \times\left[\beta J_{\nu}\left(\frac{\alpha^{1 / 2} \xi}{2}\right)+(1-\beta) Y_{\nu}\left(\frac{\alpha^{1 / 2} \xi}{2}\right)\right]^{-2} .
\end{aligned}
$$

Case $\mu=-1 / 2, n=3(\operatorname{cr}[\mathbf{7 0}]): \quad f=\frac{\alpha}{\xi^{2}} \tan ^{2}\left(\frac{\alpha^{1 / 2}}{2}(\xi-c)\right)$

or $f=\frac{\alpha}{\xi^{2}} \tanh ^{2}\left(\frac{\alpha^{1 / 2}}{2}(\xi-c)\right)$.

Case $\mu=-1 / 2, n=1: \quad f=\frac{\alpha^{2}}{\left(2-\alpha^{1 / 2} \xi \cot \left[\alpha^{1 / 2}(\xi-c) / 2\right]\right)^{2}}$

or $f=\frac{\alpha^{2}}{\left(2-\alpha^{1 / 2} \xi \operatorname{coth}\left[\alpha^{1 / 2}(\xi-c) / 2\right]\right)^{2}}$.

Case $\mu=n / 2-2: \quad f=\xi^{(2-n) /(\mu+1)} g$, where

$$
\int \frac{g^{n / 2-2}}{g+(n-2) \alpha} d g=-\frac{1}{(n-2)^{2}} \xi^{n-2} .
$$

\section{Variable coefficient diffusion-convection equations}

To obtain invariant solutions of the variable coefficient diffusion-convection equations of form (1) two approaches were used. The first one is the direct finding of solutions invariant with respect to a subalgebra of the Lie invariance algebra, and the second one is reconstructing of new solutions from the known ones using equivalence transformations.

As an example of implementation of the first approach we adduce some of the invariant solutions of equation $[\mathbf{2 4}, \mathbf{4 8}]$.

$$
|x|^{p} u_{t}=\left(|u|^{\mu} u_{x}\right)_{x}
$$


Namely,

$$
\begin{gathered}
u=\left|c_{1} x+c_{0}\right|^{1 /(\mu+1)}, \\
u=\left(\frac{-c_{1} \mu}{(p+2)(2+\mu+p+\mu p)}\right)^{1 / \mu}\left(c_{1} t+c_{0}\right)^{-1 / \mu} x^{(2+p) / \mu} \\
u=t^{(p+1) \beta}\left(\frac{\mu \beta}{2+p} x^{2+p} t^{(p+2) \beta}+c_{0}\right)^{1 / \mu}, \quad \text { where } \beta=-\frac{1}{p \mu+p+\mu+2} .
\end{gathered}
$$

For equation (22) with $p=-(\mu+2) /(\mu+1)$ solution obtained with multiplicative separation of variables is known:

$$
u=e^{-c_{1} t}\left(c_{1}(\mu+1)^{2} x^{\mu /(\mu+1)} e^{c_{1} \mu t}+c_{2}\right)^{1 / \mu}
$$

See also the next two sections for invariant solutions of essentially variable coefficient equations.

Another possible way of finding exact solutions is based on application of equivalence transformations. The complete extended equivalence group $\hat{G}^{\sim}$ of class (1) is formed by the transformations $[\mathbf{2 7}, \mathbf{2 8}]$

$$
\tilde{f}=\frac{\varepsilon_{1} \delta_{1} \varphi}{X_{x}} f, \quad \tilde{g}=\varepsilon_{1} \varepsilon_{2}^{-1} X_{x} \varphi g, \quad \tilde{h}=\varepsilon_{1} \varepsilon_{3}^{-1} \varphi h, \quad \tilde{A}=\varepsilon_{2} A, \quad \tilde{B}=\varepsilon_{3}\left(B+\varepsilon_{4} A\right),
$$

where $\delta_{j}(j=\overline{1,4})$ and $\varepsilon_{i}(i=\overline{1,4})$ are arbitrary constants, $\delta_{1} \delta_{3} \varepsilon_{1} \varepsilon_{2} \varepsilon_{3} \neq 0, X$ is an arbitrary smooth function of $x, X_{x} \neq 0, \varphi=e^{-\varepsilon_{4} \int \frac{h(x)}{g(x)} d x}$.

It appears also, that class (1) contains equations being mutually equivalent with respect to point transformations which do not belong to this group. In particular, it is proved in $[\mathbf{2 8}, \mathbf{3 1}, \mathbf{4 9}]$ that if an equation of form (1) is invariant with respect to a Lie algebra of dimension not less than 4 then it can be reduced by point transformations to a constant coefficient diffusion-convection equation (18). All such equations and corresponding transformations were found in $[\mathbf{2 8}, \mathbf{3 1}, \mathbf{4 9}]$. Some of them were known previously [41]. For the convenience of the readers we adduce the results of group classification up to the extended equivalence group (23) in Appendix A.

Up to equivalence transformations (23) the list of equations of form (1) reducible to the constant coefficient form together with corresponding transformations is exhausted by the following ones [28]:

1. $u_{t}=\left(e^{u} u_{x}\right)_{x}+\varepsilon x u_{x} \rightarrow(8): \tilde{t}=e^{2 \varepsilon t} /(2 \varepsilon), \tilde{x}=x e^{\varepsilon t}, \tilde{u}=u$;

2. $x^{-3} u_{t}=\left(e^{u} u_{x}\right)_{x} \rightarrow(8): \quad \tilde{t}=t \operatorname{sign} x, \tilde{x}=1 / x, \tilde{u}=u-\ln |x|$;

3. $x^{-3} u_{t}=\left(e^{u} u_{x}\right)_{x}+x^{-2} u_{x} \rightarrow(8): \tilde{t}=\left(e^{2 t} t \operatorname{sign} x\right) / 2, \tilde{x}=e^{-t} / x, \tilde{u}=u-t-\ln |x|$;

4. $u_{t}=\left(|u|^{\mu} u_{x}\right)_{x}+\varepsilon x u_{x} \rightarrow(9): \quad \tilde{t}=e^{2 \varepsilon t} /(2 \varepsilon), \tilde{x}=x e^{\varepsilon t}, \tilde{u}=u$;

5. $|x|^{-\frac{3 \mu+4}{\mu+1}} u_{t}=\left(|u|^{\mu} u_{x}\right)_{x} \rightarrow(9): \quad \tilde{t}=t, \tilde{x}=-1 / x, \tilde{u}=|x|^{-\frac{1}{1+\mu}} u$;

6. $|x|^{-\frac{3 \mu+4}{\mu+1}} u_{t}=\left(|u|^{\mu} u_{x}\right)_{x}+\left.\varepsilon x|x|^{-\frac{3 \mu+4}{\mu+1}} u_{x}\right|_{\mu \neq 2} \rightarrow(9): \quad \tilde{t}=\frac{\mu+1}{\varepsilon(\mu+2)}\left(1-e^{-\varepsilon \frac{\mu+2}{\mu+1} t}\right)$,

$\tilde{x}=x e^{\varepsilon t}, \tilde{u}=u$;

7. $x^{-2} u_{t}=\left(u^{-2} u_{x}\right)_{x}+\varepsilon x^{-1} u_{x} \rightarrow(5): \quad \tilde{t}=t, \tilde{x}=x e^{\varepsilon t}, \tilde{u}=u$; 
8. $e^{x} u_{t}=\left(u^{-1} u_{x}\right)_{x} \rightarrow(15): \quad \tilde{t}=t, \tilde{x}=x, \tilde{u}=e^{x} u$;

9. $e^{x} u_{t}=\left(u^{-1} u_{x}\right)_{x}+\varepsilon e^{x} u_{x} \rightarrow(15): \quad \tilde{t}=e^{\varepsilon t} / \varepsilon, \tilde{x}=x+\varepsilon t, \tilde{u}=e^{x+\varepsilon t} u$.

Combining these transformations with symmetry and equivalence transformations one can easily obtain solutions of such "non-essentially variable coefficient" equations. For instance, starting from solutions of equation (8), we obtain corresponding solutions for the more complicated and interesting equation

$$
\frac{e^{x}}{\left(\gamma e^{x}+1\right)^{3}} u_{t}=\left(e^{u} u_{x}\right)_{x}+e^{u} u_{x}
$$

having the density $f$ localized in space $[49]$ :

$$
u=\ln \left|c_{1}+c_{0}\left(e^{-x}+\gamma\right)\right|, \quad u=\ln \left(-\frac{1}{2 t\left(e^{-x}+\gamma\right)}-\frac{c_{1}}{t}+c_{0} \frac{e^{-x}+\gamma}{t}\right) .
$$

Similarly one can find exact solutions of equation

$$
e^{x} u_{t}=\left(u^{-1} u_{x}\right)_{x}+\varepsilon e^{x} u_{x}
$$

that is reducible to the fast diffusion equation [29]:

$$
\begin{gathered}
u=\frac{e^{-(x+\varepsilon t)}}{1+c e^{x+\varepsilon t+e^{\varepsilon t} / \varepsilon}}, \quad u=\frac{\varepsilon e^{-(x+\varepsilon t)}}{\varepsilon x+\varepsilon^{2} t-e^{\varepsilon t} \pm e^{\varepsilon t-\varepsilon(x+\varepsilon t) e^{-\varepsilon t}},} \\
u=\frac{2 e^{-x}}{\varepsilon(x+\varepsilon t)^{2} \pm e^{2 \varepsilon t}}, \quad u=\frac{2 e^{-x}}{\varepsilon \cos ^{2}(x+\varepsilon t)}, \quad u=-\frac{2 e^{-x}}{\varepsilon \cosh ^{2}(x+\varepsilon t)}, \\
u=\frac{2 e^{-x}}{\varepsilon \sin ^{2}(x+\varepsilon t)}, \quad u=\frac{2 e^{-(x+\varepsilon t)} \sin \left(2 e^{\varepsilon t} / \varepsilon\right)}{\cos \left(2 e^{\varepsilon t} / \varepsilon\right)-\cos 2(x+\varepsilon t)}, \\
u=\frac{2 e^{-(x+\varepsilon t)} \sinh \left(2 e^{\varepsilon t} / \varepsilon\right)}{\cosh 2(x+\varepsilon t)-\cosh \left(2 e^{\varepsilon t} / \varepsilon\right)}, \quad u=-\frac{2 e^{-(x+\varepsilon t)} \sinh \left(2 e^{\varepsilon t} / \varepsilon\right)}{\cosh 2(x+\varepsilon t)+\cosh \left(2 e^{\varepsilon t} / \varepsilon\right)}, \\
u=\frac{2 e^{-(x+\varepsilon t)} \cosh \left(2 e^{\varepsilon t} / \varepsilon\right)}{\sinh 2(x+\varepsilon t)-\sinh \left(2 e^{\varepsilon t} / \varepsilon\right)}, \quad u=\frac{2 e^{-(x+\varepsilon t)} \sin \left(2 e^{\varepsilon t} / \varepsilon\right)}{\cosh 2(x+\varepsilon t)-\cos \left(2 e^{\varepsilon t} / \varepsilon\right)}, \\
u=\frac{2 e^{-(x+\varepsilon t)} \sinh \left(2 e^{\varepsilon t} / \varepsilon\right)}{\cosh \left(2 e^{\varepsilon t} / \varepsilon\right)-\cos 2(x+\varepsilon t)} .
\end{gathered}
$$

Using the same approach we constructed exact solutions for the following equations $[29,31,49]$ :

$$
\begin{aligned}
& e^{-2 x+\gamma e^{-x}} u_{t}=\left(u^{-1} u_{x}\right) x+u^{-1} u_{x}: \\
& u=c_{0} e^{\left(c_{1}-\gamma\right) e^{-x}}, \quad u=\frac{2 c_{1}^{2} t e^{-\gamma e^{-x}}}{\cos ^{2} c_{1}\left(e^{-x}+c_{0}\right)}, \quad u=\frac{2 t c_{0} c_{1}^{2} e^{\left(c_{1}-\gamma\right) e^{-x}}}{\left(1-c_{0} e^{c_{1} e^{-x}}\right)^{2}}, \\
& u=\frac{c_{1} e^{-\gamma e^{-x}}}{-\varepsilon+c_{0} e^{c_{1}\left(e^{-x}-\varepsilon t\right)}}, \quad u=\frac{\varepsilon e^{-\gamma e^{-x}}}{e^{-x}-\varepsilon t+c_{0}}, \quad u=\frac{2 t e^{-\gamma e^{-x}}}{\left(e^{-x}+c_{1}\right)^{2}+c_{0} t^{2}} .
\end{aligned}
$$

Equation

$$
\frac{e^{-2 x}}{\left(e^{-x}+\gamma\right)^{\frac{4+3 \mu}{1+\mu}}} u_{t}=\left(u^{\mu} u_{x}\right)_{x}+u^{\mu} u_{x}
$$


has exact solutions of the form

$$
\begin{gathered}
u=\left|c_{0}\left(e^{-x}+\gamma\right)-c_{1}\right|^{\frac{1}{\mu+1}}, \quad u=\left(c_{0}+\frac{\varepsilon \mu}{e^{-x}+\gamma}+\varepsilon^{2} \mu t\right)^{\frac{1}{\mu}}\left|e^{-x}+\gamma\right|^{-\frac{1}{\mu+1}} \\
u=\left(-\frac{\mu}{\mu+2} \frac{1}{2 t}\left(c_{0}-\frac{1}{e^{-x}+\gamma}\right)^{2}+c_{1}|t|^{-\frac{\mu}{\mu+2}}\right)^{\frac{1}{\mu}}\left|e^{-x}+\gamma\right|^{-\frac{1}{\mu+1}} \\
u=\left(-\frac{\mu}{\mu+2} \frac{1}{2 t}\left(c_{0}-\frac{1}{e^{-x}+\gamma}\right)^{2}+\right. \\
\left.+c_{1}\left(c_{0}-\frac{1}{e^{-x}+\gamma}\right)^{\frac{\mu}{\mu+1}}|t|^{-\frac{\mu(2 \mu+3)}{2(\mu+1)^{2}}}\right)^{\frac{1}{\mu}}\left|e^{-x}+\gamma\right|^{-\frac{1}{\mu+1}} \\
u=\left(6 t+c_{1}^{\prime}+c_{2} e^{-x}\right)^{2}\left(e^{-x}+\gamma\right)^{6} . \\
\boldsymbol{e}^{\boldsymbol{x}} \boldsymbol{u}_{\boldsymbol{t}}=\left(\boldsymbol{u}^{-\boldsymbol{1}} \boldsymbol{u}_{\boldsymbol{x}}\right)_{\boldsymbol{x}}+\boldsymbol{\mu} \boldsymbol{e}^{\boldsymbol{x}} \boldsymbol{u}_{\boldsymbol{x}}
\end{gathered}
$$

admits the following invariant exact solutions:

$$
\begin{gathered}
u=\frac{c_{0}}{\mu} e^{\left(1-c_{1}\right)(x+\mu t)}, \quad u=\frac{2 c_{1}^{2}}{\mu} \frac{e^{-x}}{\cos ^{2}\left[c_{1}\left(x+\mu t+c_{0}\right)\right]}, \\
u=\frac{2 c_{0} c_{1}^{2}}{\mu} \frac{e^{\left(c_{1}-1\right) x+c_{1} \mu t}}{\left[1-c_{0} e^{c_{1}(x+\mu t)}\right]^{2}}, \quad u=\frac{c_{1}}{\mu} \frac{e^{-(x+\mu t)}}{c_{2}+c_{0} e^{c_{1}\left(x+\mu t+c_{2} e^{\mu t}\right)}}, \\
u=\frac{2}{\mu} \frac{e^{-x}}{\left(x+\mu t+c_{1}\right)^{2}+c_{0} e^{2 \mu t}}, \quad u=\frac{1}{\mu} \frac{e^{-(x+\mu t)}}{x+\mu t-e^{\mu t}+c_{0}} . \\
\boldsymbol{f}(\boldsymbol{x}) \boldsymbol{u}_{\boldsymbol{t}}=\left(\boldsymbol{u}^{-\mathbf{1}} \boldsymbol{u}_{\boldsymbol{x}}\right)_{\boldsymbol{x}}-\left(\frac{\boldsymbol{f}_{\boldsymbol{x}}}{\mathbf{2 f}}-\frac{\sqrt{\boldsymbol{f}}}{\int \sqrt{\boldsymbol{f}} \boldsymbol{d} \boldsymbol{x}}\right) \boldsymbol{u}^{-\mathbf{1}} \boldsymbol{u}_{\boldsymbol{x}},
\end{gathered}
$$

where $f=f(x)$ is an arbitrary positive function has the following invariant solutions:

$$
\begin{gathered}
u=c_{0}\left(\int \sqrt{f} d x\right)^{c_{1}-2}, \quad u=\frac{2 c_{1}^{2} t\left(\int \sqrt{f} d x\right)^{-2}}{\cos ^{2}\left[c_{1}\left(\ln \int \sqrt{f} d x+c_{0}\right)\right]}, \\
u=\frac{2 c_{0} c_{1}^{2} t\left(\int \sqrt{f} d x\right)^{c_{1}-2}}{\left[1-c_{0}\left(\int \sqrt{f} d x\right)^{c_{1}}\right]^{2}}, \quad u=\frac{c_{1}\left(\int \sqrt{f} d x\right)^{-2}}{c_{2}+c_{0}\left(\int \sqrt{f} d x\right)^{c_{1}} e^{c_{1} c_{2} t}}, \\
u=\frac{\left(\int \sqrt{f} d x\right)^{-2}}{\ln \left(\int \sqrt{f} d x\right)-t+c_{0}}, \quad u=\frac{2 t\left(\int \sqrt{f} d x\right)^{-2}}{\left[\ln \left(\int \sqrt{f} d x\right)+c_{1}\right]^{2}+c_{0} t^{2}} . \\
\boldsymbol{u}_{\boldsymbol{t}}=\left(\boldsymbol{u}^{-\mathbf{1}} \boldsymbol{u}_{\boldsymbol{x}}\right)_{\boldsymbol{x}}+\boldsymbol{\mu} \boldsymbol{x} \boldsymbol{u}_{\boldsymbol{x}}
\end{gathered}
$$

admits the following solutions:

$$
\begin{gathered}
u=c_{0} e^{c_{1} x e^{\mu t}}, \quad u=\frac{c_{1}^{2} e^{2 \mu t}}{\mu \cos ^{2}\left[c_{1}\left(x e^{\mu x}+c_{0}\right)\right]}, \quad u=\frac{c_{0} c_{1}^{2} e^{\left(2 \mu t+c_{1} x e^{\mu t}\right)}}{\mu\left(1-c_{0} e^{c_{1} x e^{\mu t}}\right)^{2}}, \\
u=\frac{c_{1}}{c_{2}+c_{0} e^{c_{1}\left(x e^{\mu t}+\frac{c_{2}}{2 \mu} e^{2 \mu t}\right)}}, \quad u=\frac{2 \mu}{2 \mu x e^{\mu t}-e^{2 \mu t}+2 \mu c_{0}} \\
u=\frac{4 \mu e^{2 \mu t}}{4 \mu^{2}\left(x e^{\mu t}+c_{1}\right)^{2}+c_{0} e^{4 \mu t}} .
\end{gathered}
$$


See $[\mathbf{2 8}, \mathbf{2 9}, \mathbf{3 1}, \mathbf{4 9}]$ for more detail and more examples. Since the solutions of these equations can be reconstructed from ones presented in other sections, we tern back to the more interesting cases, in particular, to the equations which are "essentially variable coefficient".

\section{Examples of Lie reduction of variable coefficient equation}

The results of this section have been obtained in $[\mathbf{2 9}, \mathbf{3 1}]$.

In this section we consider Lie reductions of some variable coefficient equations of form (1). We start from the equation

$$
e^{p x} u_{t}=\left[e^{u} u_{x}\right]_{x}+e^{p x} u_{x}
$$

which admits three-dimensional Lie algebra $Q_{1}=-p^{-1} \partial_{t}, Q_{2}=e^{-p t}\left(\partial_{t}-\partial_{x}\right)$, $Q_{3}=\partial_{x}+p \partial_{u}$. The only non-zero commutators of these operators are $\left[Q_{1}, Q_{2}\right]=$ $Q_{2}$. Therefore $A^{\max }$ is a realization of the algebra $2 A_{2.1}[\mathbf{4 0}]$. All the possible inequivalent (with respect to inner automorphisms) one-dimensional subalgebras of $A_{2.1} \oplus A_{1}$ [46] are exhausted by the ones listed in Table 11 along with the corresponding ansätze and the reduced odes.

Table 11. Reduced odes for (24). $\lambda \neq 0, \varepsilon= \pm 1$

\begin{tabular}{|l|l|c|c|l|}
\hline $\mathrm{N}$ & Subalgebra & Ansatz $u=$ & $\omega$ & \multicolumn{1}{|c|}{ Reduced ODE } \\
\hline 1 & $Q_{1}$ & $\varphi(\omega)$ & $x$ & $\left(e^{\varphi} \varphi^{\prime}\right)^{\prime}+e^{p x} \varphi^{\prime}=0$ \\
2 & $Q_{2}$ & $\varphi(\omega)$ & $t+x$ & $\left(e^{\varphi} \varphi^{\prime}\right)^{\prime}=0$ \\
3 & $Q_{3}$ & $\varphi(\omega)+p x$ & $t$ & $\varphi^{\prime}=p^{2} e^{\varphi}+p$ \\
4 & $Q_{1}+\lambda Q_{3}$ & $\varphi(\omega)+\lambda p t$ & $x-\lambda t$ & $e^{\omega}\left[\lambda p-(\lambda+1) \varphi^{\prime}\right]=\left(e^{\varphi} \varphi^{\prime}\right)^{\prime}$ \\
5 & $Q_{2}+\varepsilon Q_{3}$ & $\varphi(\omega)+\varepsilon e^{p t}$ & $x+t-\frac{\varepsilon}{p} e^{p t}$ & $e^{p \omega} \varepsilon\left(p-\varphi^{\prime}\right)=\left(e^{\varphi} \varphi^{\prime}\right)^{\prime}$ \\
\hline
\end{tabular}

As a second example we consider the equation

$$
\boldsymbol{x}^{p} \boldsymbol{u}_{\boldsymbol{t}}=\left[\boldsymbol{u}^{m} \boldsymbol{u}_{\boldsymbol{x}}\right]_{\boldsymbol{x}}+\boldsymbol{x}^{p+1} \boldsymbol{u}_{\boldsymbol{x}}
$$

The invariance algebra of (25) is generated by the operators $Q_{1}=-(p+2)^{-1} \partial_{t}$, $Q_{2}=e^{-(p+2) t}\left(\partial_{t}-x \partial_{x}\right), Q_{3}=m x \partial_{x}+(p+2) u \partial_{u}$ and is a realization of the algebra $A_{2.1} \oplus A_{1}$ too. The reduced equations for (25) are listed in table 12.

Table 12. Reduced odes for (25). $\lambda \neq 0, \varepsilon= \pm 1$

\begin{tabular}{|l|l|c|c|l|}
\hline $\mathrm{N}$ & Subalgebra & Ansatz $u=$ & $\omega$ & \multicolumn{1}{c|}{ Reduced ODE } \\
\hline 1 & $Q_{1}$ & $\varphi(\omega)$ & $x$ & $\left(\varphi^{m} \varphi^{\prime}\right)^{\prime}+x^{p+1} \varphi^{\prime}=0$ \\
2 & $Q_{2}$ & $\varphi(\omega)$ & $x e^{t}$ & $\left(\varphi^{m} \varphi^{\prime}\right)^{\prime}=0$ \\
3 & $Q_{3}$ & $x^{\frac{p+2}{m}} \varphi(\omega)$ & $t$ & $\varphi^{\prime}=\frac{(p+2)(p+2+m)}{m^{2}} \varphi^{m+1}+\frac{p+2}{m} \varphi$ \\
4 & $Q_{1}+\lambda Q_{3}$ & $e^{\lambda(p+2) t} \varphi(\omega)$ & $x e^{-\lambda m t}$ & $\left(\varphi^{m} \varphi^{\prime}\right)^{\prime}+(1+\lambda m) \omega^{p+1} \varphi^{\prime}$ \\
& & & & $=\lambda(p+2) \omega^{p} \varphi$ \\
5 & $Q_{2}+\varepsilon Q_{3}$ & $e^{\varepsilon e^{(p+2) t}} \varphi(\omega)$ & $x e^{t-\frac{m \varepsilon}{p+2} e^{(p+2) t}}$ & $\left(\varphi^{m} \varphi^{\prime}\right)^{\prime}=\varepsilon(p+2) \omega^{p} \varphi$ \\
& & & & $-m \varepsilon \omega^{p+1} \varphi^{\prime}$ \\
\hline
\end{tabular}

At last, let us analyze in more detail equation

$$
e^{p x^{2}} u_{t}=\left(e^{p x^{2}} u_{x}\right)_{x}+e^{p x^{2}} u u_{x}
$$

which is invariant with respect to three-dimensional Lie symmetry algebra

$$
\left\langle\partial_{t}, e^{-2 p t} \partial_{x}, \partial_{x}-2 p \partial_{u}\right\rangle
$$

In contrast to the case of equations with four-dimensional Lie symmetry algebra we cannot reduce equation (26) to a constant coefficient equation of form (1). However, it is an interesting feature of this equation that using a point transformation $v=$ 
$u+2 p x$ it can be mapped to a constant coefficient reaction-convection-diffusion equation

$$
v_{t}=v_{x x}+v v_{x}-2 p v
$$

that does not belong to class (1). For simplification of the technical calculations we will investigate the constant coefficient equation (27) instead of (26). The Lie symmetry algebra of equation (27)

$$
\left\langle X_{1}=\partial_{t}, X_{2}=e^{-2 p t}\left(\partial_{x}+2 p \partial_{v}\right), X_{3}=\partial_{x}\right\rangle
$$

is a realization of $A_{2.1} \oplus A_{1}$ [40]. These operators generate the following group of point transformations:

$$
\tilde{t}=t+\varepsilon_{1}, \quad \tilde{x}=x+\varepsilon_{2} e^{-2 p t}+\varepsilon_{3}, \quad \tilde{v}=v+2 \varepsilon_{2} p e^{-2 p t} .
$$

A list of proper inequivalent subalgebras of the given algebra is exhausted by the following ones

$$
\left\langle X_{1}+\alpha X_{3}\right\rangle, \quad\left\langle X_{3}+\varepsilon X_{2}\right\rangle, \quad\left\langle X_{2}\right\rangle, \quad\left\langle X_{1}, X_{3}\right\rangle, \quad\left\langle X_{2}, X_{3}\right\rangle, \quad\left\langle X_{1}+\beta X_{3}, X_{2}\right\rangle,
$$

where $\alpha$ and $\beta$ are arbitrary constants, $\varepsilon=0, \pm 1[\mathbf{4 6}]$.

The first three (one-dimensional) subalgebras lead to Lie reductions to ordinary differential equations, the fourth and sixth (two-dimensional) ones yield reductions to algebraic equations. Lie reductions with respect to these subalgebras are summarized in Table 13. One can easily check that it is impossible to construct a Lie ansatz corresponding to the subalgebra $\left\langle X_{2}, X_{3}\right\rangle$.

Table 13. Lie reductions of equation (27).

\begin{tabular}{|l|l|c|c|l|}
\hline $\mathrm{N}$ & Subalgebra & Ansatz $v=$ & $\omega$ & \multicolumn{1}{|c|}{ Reduced equation } \\
\hline 1 & $\left\langle X_{1}+\alpha X_{3}\right\rangle$ & $\varphi(\omega)$ & $x-\alpha t$ & $\varphi^{\prime \prime}+(\varphi+\alpha) \varphi^{\prime}-2 p \varphi=0$ \\
2 & $\left\langle X_{3}+\varepsilon X_{2}\right\rangle$ & $\varphi(\omega)+\frac{2 p \varepsilon x}{e^{2 p t}+\varepsilon}$ & $t$ & $\varphi^{\prime}=\frac{2 p e^{2 p \omega}}{e^{2 p \omega}+\varepsilon} \varphi$ \\
3 & $\left\langle X_{2}\right\rangle$ & $\varphi(\omega)+2 p x$ & $t$ & $\varphi^{\prime}=0$ \\
4 & $\left\langle X_{1}, X_{3}\right\rangle$ & $C$ & - & $C=0$ \\
5 & $\left\langle X_{1}+\beta X_{3}, X_{2}\right\rangle$ & $2 p x-2 p \beta t+C$ & - & $-2 p \beta=0$ \\
\hline
\end{tabular}

Integration of equations $2-5$ from Table 13 give the following invariant solutions of equation (27):

$$
v=0, \quad v=2 p x+C, \quad v=\frac{2 p \varepsilon x+C e^{2 p t}}{e^{2 p t}+\varepsilon} .
$$

The corresponding exact invariant solutions of equation (26) have the form

$$
u=-2 p x, \quad u=C, \quad u=\frac{2 p \varepsilon x+C e^{2 p t}}{e^{2 p t}+\varepsilon}-2 p x,
$$

where $C$ is an arbitrary constant.

Ansatzes 4.2 and 4.3 give a hint for a possible form

$$
v=\varphi(t) x+\psi(t)
$$

of nonlinear separation of variables for construction of exact solutions of equation (27). Substitution of the ansatz to equation (27) leads to antireduction:

$$
\varphi^{\prime}=\varphi^{2}-2 p \varphi, \quad \psi^{\prime}=\varphi \psi-2 p \psi .
$$

Solving the above system of ODEs for $\varphi$ and $\psi$ we obtain exactly the solutions of equations 4.2 and 4.3 . 
Note 1. Using the point transformation $\tilde{t}=e^{-2 p t}, \tilde{x}=x, \tilde{v}=e^{2 p t} v$ equation (27) can be mapped to a variable coefficient Burgers equation $\tilde{v}_{\tilde{t}}=-2 p \tilde{t}^{-1} \tilde{v}_{\tilde{x} \tilde{x}}-$ $2 p \tilde{v} \tilde{v}_{\tilde{x}}$ studied in [37].

Note 2. The well-known Cole-Hopf transformation $v=2 w_{x} / w$ reduces equation (27) to the famous constant coefficient reaction-diffusion equation with weak nonlinearity

$$
w_{t}=w_{x x}-2 p w \ln |w| .
$$

After application of the Cole-Hopf transformation to the list of known exact solutions (see, e.g., [48]) of the equation with weak nonlinearity we obtain exactly solutions (28) of equation (27).

\section{Exact solutions of $\operatorname{sl}(2, \mathbb{R})$-invariant equation}

Analyzing the results of group classification of diffusion-convection equations, we can observe a number of $\hat{G}^{\sim}$-inequivalent equations (1) which are invariant with respect to different realizations of the algebra $\operatorname{sl}(2, \mathbb{R})$. The set of such equations is practically exhausted by the well-known ("constant coefficient") Burgers and $u^{-4 / 3}$. diffusion equations and by the equations which are equivalent to them with respect to additional transformations. This set is supplemented by the unique essentially variable coefficient equation $[\mathbf{2 8 , 3 1}]$

$$
x^{2} u_{t}=\left(u^{-6 / 5} u_{x}\right)_{x}+x^{2} u_{x}
$$

$\operatorname{sl}(2, \mathbb{R})$-invariance of $(29)$ is directly connected with the fact that $h$ is not constant. The corresponding realization

$$
P_{t}=\partial_{t}, \quad D=2 t \partial_{t}+2 x \partial_{x}-5 u \partial_{u}, \quad \Pi=t^{2} \partial_{t}+\left(2 t x+x^{2}\right) \partial_{x}-5(t+x) u \partial_{u} .
$$

of the algebra $\operatorname{sl}(2, \mathbb{R})$ is quite different from ones of cases of Burgers and $u^{-4 / 3}$. diffusion equations and is the maximal Lie invariance algebra of equation (29). It was a reason to study equation (29) from the symmetry point of view in detail in $[29]$. These operators generate the following one-parameter groups of point transformations:

$$
\begin{gathered}
P_{t}: \quad \tilde{t}=t+\varepsilon, \quad \tilde{x}=x, \quad \tilde{u}=u ; \\
D: \quad \tilde{t}=e^{\varepsilon} t, \quad \tilde{x}=e^{\varepsilon} x, \quad \tilde{v}=e^{3 \varepsilon} v ; \\
\Pi: \quad \tilde{t}=\frac{t}{1-\varepsilon t}, \quad \tilde{x}=\frac{t+x}{1-\varepsilon(t+x)}-\frac{t}{1-\varepsilon t}, \quad \tilde{u}=(1-\varepsilon(t+x))^{6} u .
\end{gathered}
$$

The complete Lie invariance group $G^{\max }$ is generated by both the above continuous transformations and the discrete transformation of changing of sign in the triple $(t, x, u)$. The transformations from $G^{\max }$ can be used for construction of new solutions from known ones.

A list of proper $G^{\text {max }}$-inequivalent subalgebras of $A^{\max }$ is exhausted by the algebras $\left\langle P_{t}\right\rangle,\langle D\rangle,\left\langle P_{t}+\Pi\right\rangle,\left\langle P_{t}, D\right\rangle$. Reduction of (29) with respect to these subalgebras and application of the invariance transformations lead to the following 
set of $G^{\text {max }}$-inequivalent Lie invariant exact solutions (below $\delta \in\{0,1\}$ ):

$$
\begin{gathered}
u=C(t+x)^{-5}, \quad u=2^{-5 / 6}\left(\frac{x}{t}\right)^{-5 / 2}(t+x)^{-5 / 2}, \\
u=\left(\frac{3 x^{4}}{4 t} \frac{(t+x)^{2}}{C t-1}+2 \frac{x^{3}}{t^{3}}(t+x)^{3}\right)^{-5 / 6}, \\
u=x^{-5 / 6}\left(\frac{(t+x)^{2}}{C t+1}\right)^{-5 / 6}\left(\frac{5}{4} \frac{x^{3}}{t^{3}}+2 \frac{x^{2}}{t^{2}}(C(t+x)+1)\right)^{-5 / 6} .
\end{gathered}
$$

In $[\mathbf{2 9}]$ it was proposed to use functional separation of variables

$$
u=\left(\sum_{i=0}^{6} \varphi^{i}(t) x^{i}\right)^{-5 / 6} .
$$

to obtain solutions of equation (29). The set of all solutions of the form (30) is closed with respect to transformations from $G^{\max }$ and is exhausted, up to translations with respect to $t$ and scale transformations, by the above solutions $u=\delta$ and $u=\delta(t+x)^{-5}$ and the solutions given by the generalized ansatz

$$
u=\left(2 x^{3}+\varphi^{4}(t) x^{4}+\varphi^{5}(t) x^{5}+\varphi^{6}(t) x^{6}\right)^{-5 / 6}
$$

and the corresponding reduced system

$$
\varphi_{t}^{4}=7 \varphi^{5}-\frac{4}{3}\left(\varphi^{4}\right)^{2}, \quad \varphi_{t}^{5}=18 \varphi^{6}-\frac{4}{3} \varphi^{4} \varphi^{5}, \quad \varphi_{t}^{6}=-\frac{5}{6}\left(\varphi^{5}\right)^{2}+2 \varphi^{4} \varphi^{6} .
$$

System (32) can be reduced to the single third-order ordinary differential equation on the function $\varphi^{4}$ :

$$
63 \varphi_{t t t}^{4}+387\left(\varphi_{t}^{4}\right)^{2}+126 \varphi^{4} \varphi_{t t}^{4}+192\left(\varphi^{4}\right)^{2} \varphi_{t}^{4}+16\left(\varphi^{4}\right)^{4}=0
$$

having particular solutions $\varphi^{4}=0, \varphi^{4}=C / t$, where $C \in\{0,3 / 4,21 / 4,6\}$, that lead to Lie invariant solutions of (29).

Conclusive remarks. This work is constantly under updating. So, the author will appreciate any suggestions, comments and references sent to the e-mail ivanova@imath.kiev.ua.

Acknowledgment. The author is grateful to G.W. Bluman, V.M. Boyko, V.I. Lahno, O.I. Morozov, A.G. Nikitin, R.O. Popovych, A.G. Sergyeyev, C. Sophocleous, O.O. Vaneeva, I.A. Yehorchenko, O.Yu. Zhalij for fruitful discussions and suggested references. She acknowledges the hospitality and financial support of the Department of Mathematics of the University of British Columbia and Department of Mathematics and Statistics of the University of Cyprus where the part of research has been made.

\section{References}

[1] Abramowitz M.J. and Stegun I., Handbook on mathematical functions, National Bureau of Standards Appl. Math. Series, No. 55, U.S. Govt. Printing Office, Washington, D.C., 1970.

[2] Akhatov I.Sh., Gazizov R.K., Ibragimov N.Kh., Nonlocal symmetries. A heuristic approach Itogi Nauki i Tekhniki, Current problems in mathematics. Newest results, 1989, V.34, 3-83, (Russian, translated in J. Soviet Math. 199155 N 1 1401-1450)

[3] Amerov T.K., On conditional invariance of nonlinear heat equation, Theoretical and algebraic analysis of equations of mathematical physics (Kiev: Institute of mathematics), 1990, 12-14. 
[4] Anderson R.L. and Ibragimov N.H., Lie-Bäcklund transformations in applications, Philadalphia, Pensilvania, SIAM, 1979.

[5] Aristov S.N., Exact periodic and localized solutions of the equation $h_{t}=\Delta \ln h, J$. Appl. Mech. \& Tech. Phys., 1999, V.40, No. 1, 16-19.

[6] Barenblatt G.I., On nonsteady motions of gas and fluid in porous medium, Appl. Math. and Mech., 1952, V.16, No.1, 67-78.

[7] Barenblatt G.I. and Zel'dovich Ya.B., On stability of flame-propagation, Prikl. Mat. Meh., 1957, V.21, 856-859 (Russian).

[8] Bluman G. and Anco S.C., Symmetry and integration methods for differential equations, Applied Mathematical Sciences, V.154, New-York, Springer-Verlag, 2002.

[9] Bluman G.W. and Cole J.D., The general similarity solution of the heat equation, J. of Math. and Mech., 1969, 18, N 11, 1025-1042.

[10] Bluman G. and Kumei S., On the remarkable nonlinear diffusion equation $(\partial / \partial x)[a(u+$ $\left.b)^{-2}(\partial u / \partial x)\right]-\partial u / \partial t=0, J$. Math. Phys., 1980, V.21, N 5, 1019-1023.

[11] Bluman G.W., Kumei S., Symmetries and Differential Equations, New York, Springer, 1989.

[12] Bluman G.W., Reid G.J. and Kumei S., New classes of symmetries for partial differential equations, J. Math. Phys., 1988, V.29, 806-811.

[13] Boussinesq J., Recherches théoriques sur l'écoulement des nappes infiltrées dans le sol; compléments, J. Math. Pure Appl., 1904, V.10 (1), 5-78.

[14] Cole J.D., On a quasilinear parabolic equation used in aerodinamics, Quart. Appl. Math., 1951, V.9, 225-236.

[15] Dorodnitsyn V.A. On invariant solutions of non-linear heat equation with a sourse, Zhurn. Vych. Matemat. Matemat. Fiziki, 1982, V.22, N 6, 1393-1400 (in Russian).

[16] Doyle Ph.W. and Vassiliou P.J., Separation of variables for the 1dimensional nonlinear diffusion equation, Int. J. NonLinear Mech., 1998, V.33, 315-326.

[17] Edwards M.P. Classical symmetry reductions of nonlinear diffusion-convection equations, Phys. Lett. A., 1994, V.190, 149-154.

[18] Fokas A.S., Yortsos Y.C., On the exactly solvable equation $S_{t}=\left[(\beta S+\gamma)^{-2} S_{x}\right]_{x}+\alpha(\beta S+$ $\gamma)^{-2} S_{x}$ occurring in two-phase flow in porous media, SIAM J. Appl. Math., 1982, V.42, $318-332$.

[19] Forsyth A.R., The theory of differential equations, V.6, Cambridge, Cambridge University Press, 1906.

[20] Fushchych W.I., Serov M.I. and Amerov T.K., On nonlocal ansätzes for a nonlinear onedimensional heat equation, Proc. Acad. Sci. of Ukraine, 1992, N 1, 26-30.

[21] Fushchych W.I., Shtelen W.M., Serov M.I. and Popovych R.O., Q-conditional symmetry of the linear heat equation, Proc. Acad. of Sci. Ukraine, 1992, N 12, 28-33.

[22] Galaktionov V. A., Invariant subspaces and new explicit solutions to evolution equations with quadratic nonlinearities, Proc. Roy. Soc. Edinburgh, 1995, V.125A, 225-448.

[23] Gandarias M.L., New symmetries for a model of fast diffusion, Phys. Let. A, 2001, V.286, $153-160$.

[24] Hill J.M., Similarity solutions for nonlinear diffusion - a new integration procedure, J. Eng. Math, 1989, V.23, 141-155.

[25] Hopf E., The partial differential equation $u_{t}+u u_{x}=\mu u_{x x}$, Comm. Pure Appl. Math., 1950, V.3, 201-230.

[26] Ibragimov N.H. (Editor), Lie group analysis of differential equations - symmetries, exact solutions and conservation laws, V.1, Boca Raton, FL, CRC Press, 1994.

[27] Ivanova N.M., Popovych R.O. and Sophocleous C., Conservation laws of variable coefficient diffusion-convection equations, Proceedings of Tenth International Conference in Modern Group Analysis, (Larnaca, Cyprus, 2004), 107-113.

[28] Ivanova N.M., Popovych R.O. and Sophocleous C., Group analysis of variable coefficient diffusion-convection equations. I. Enhanced group classification 2007, to appear (arXiv:0710.2731).

[29] Ivanova N.M., Popovych R.O. and Sophocleous C., Group analysis of variable coefficient diffusion-convection equations. II. Contractions and exact solutions, 2007, to appear (arXiv:0710.3049).

[30] Ivanova N.M., Popovych R.O. and Sophocleous C., Group analysis of variable coefficient diffusion-convection equations. IV. Potential symmetries, 2007, to appear (arXiv:0710.4251). 
[31] Ivanova N.M. and Sophocleous C., On the group classification of variable coefficient nonlinear diffusion-convection equations, J. Comp. and Appl. Math., 2006, V.197, 322-344.

[32] Katkov V.L., Group classification of solutions of Hopf's equations, Zh. Prikl. Mekh. Tech. Fiz., 1965, V.6, 105-106 (in Russian).

[33] Katkov V.L., Exact solutions of some convection problems, Prikl. Mat. i Tech., V.3, 482-486.

[34] King J.R., Exact similarity solutions to some nonlinear diffusion equations, J. Phys. A: Math. Gen., 1990, V.23, 3681-3697.

[35] King J.R., Exact results for the nonlinear diffusion equations $\frac{\partial u}{\partial t}=\frac{\partial}{\partial x}\left(u^{-4 / 3} \frac{\partial u}{\partial x}\right)$ and $\frac{\partial u}{\partial t}=\frac{\partial}{\partial x}\left(u^{-2 / 3} \frac{\partial u}{\partial x}\right)$, J. Phys. A: Math. Gen., 1991, V.24, 5721-5745.

[36] King J.R., Some non-self-similar solutions to a nonlinear diffusion equation, J. Phys. A: Math. Gen., 1992, V.25, 4861-4868.

[37] Kingston J.G. and Sophocleous C., On point transformations of a generalised Burgers equation, Phys. Let. A, 1991, V.155, 15-19.

[38] Lisle I.G., Equivalence transformations for classes of differential equations, Thesis, University of British Columbia, 1992 (http://www.ise.canberra.edu.au/mathstat/StaffPages/LisleDissertation.pdf). $\quad$ (See also Lisle I.G. and Reid G.J., Symmetry classification using invariant moving frames, ORCCA Technical Report TR-00-08 (University of Western Ontario), http://www.orcca.on.ca/TechReports/2000/TR-00-08.html.)

[39] Miller W., Symmetry and Separation of Variables, Reading, Addison-Wesley, 1977.

[40] Mubarakzyanov G.M., On solvable Lie algebras, Izv. Vys. Ucheb. Zaved. Matematika, 1963, N 1 (32), 114-123 (in Russian).

[41] Munier A., Burgan, J.R., Gutierres J., Fijalkow E. and Feix M. R., Group transformations and the nonlinear heat diffusion equation, SIAM J. Appl. Math., 1981, V.40, 191-207

[42] Olver P., Applications of Lie groups to differential equations, New-York, Springer-Verlag, 1986.

[43] Oron A. and Rosenau P., Some symmetries of the nonlinear heat and wave equations, Phys. Lett. A, 1986, V.118, 172-176.

[44] Ovsiannikov L.V., Group properties of nonlinear heat equation, Dokl. AN SSSR, 1959, V.125, N 3, 492-495 (in Russian).

[45] Ovsiannikov L.V., Lectures on theory of group properties of differential equations, Novosibirsk, 1966 (in Russian).

[46] Patera J. and Winternitz P., Subalgebras of real three- and four-dimensional Lie algebras, J. Math. Phys., 1977, V.18, 1449-1455.

[47] Pattle R.E., Diffusion from an instantaneous point source with a concentration-dependent coefficient, J. Mech. Appl. Math., 1959, V.12, 407-409.

[48] Polyanin A.D. and Zaitsev V.F., Handbook of nonlinear partial differential equations, Chapman \& Hall/CRC, Boca Raton, FL, 2004.

[49] Popovych R.O. and Ivanova N.M., New results on group classification of nonlinear diffusionconvection equations, J. Phys. A: Math. Gen., 2004, V.37, 7547-7565 (math-ph/0306035).

[50] Popovych R.O. and Ivanova N.M., Potential equivalence transformations for nonlinear diffusion-convection equations, J. Phys. A: Math. Gen., 2005, V.38, 3145-3155 (math$\mathrm{ph} / 0402066)$.

[51] Popovych R.O., Vaneeva O.O. and Ivanova N.M., Potential nonclassical symmetries and solutions of fast diffusion equation, Phys. Let. A, 2007, V. 362, 166-173 (math-ph/0506067).

[52] Pukhnachov V.V., Exact multidimensional solutions of the nonlinear diffusion equation, $J$. Appl. Mech. \& Tech. Phys., 1995, V.36, 169-176.

[53] Pukhnachov V.V., Nonlocal symmetries in nonlinear heat equations, Energy methods in continuum mechanics (Oviedo, 1994), 1996, 75-99, (Dordrecht: Kluwer Acad. Publ.).

[54] Qin M., Mei F. and Fan G., New explicit solutions of the Burgers equation, Nonlinear Dyn., 2007, V.48, 91-96.

[55] Qu C., Exact solutions to nonlinear diffusion equations obtained by a generalized conditional symmetry method, IMA J. Appl. Math., 1999, V.62, 283-302.

[56] Rosenau P., Fast and superfast diffusion processes, Phys. Rev. Lett., 1995, V.74, 1056-1059.

[57] Rudykh G.A. and Semenov E.I., On new exact solutions of a onedimensional nonlinear diffusion equation with a source (sink), Zhurn. Vychisl. Matem. i Matem. Fiziki, 1998, V.38, 971-977 (in Russian). 
[58] Samarskii A.A., Galaktionov V.A., Kurdyumov S.P. and Mikhailov A.P., Blowup in problems for quasilinear parabolic equations, Berlin, Walter de Gruyter, 1995.

[59] Sokolov Yu.D., On some particular solutions of Boussinesq's equation, Ukrain. Mat. Z., 1956, V.8, 54-58 (in Russian).

[60] Sophocleous C., Potential symmetries of nonlinear diffusion-convection equations, J. Phys. A: Math. Gen, 1996, V.29, 6951-6959.

[61] Sophocleous C., Potential symmetries of inhomogeneous nonlinear diffusion equations, Bull. Austral. Math. Soc., 2000, V.61, 507-521.

[62] Sophocleous C., Classification of potential symmetries of generalised inhomogeneous nonlinear diffusion equations, Physica A 2003 V.320, 169-183.

[63] Storm M. L., Heat conduction in simple metals, J. Appl. Phys., 1951, V.22, 940-951.

[64] Strampp W., Backlund transformations for diffusion equations, Physica D, 1982, V.6, 113118.

[65] Tsyfra I., Messina A., Napoli A. and Tretynyk V., On applications of non-point and discrete symmetries for reduction of the evolution-type equations, in Proceedings of the Fifth International Conference "Symmetry in Nonlinear Mathematical Physics", Proceedings of Institute of Mathematics, Kyiv, 2004, V.50, Part 1, 271-276.

[66] Tychynin V., Petrova O. and Tertyshnyk O. Nonlocal symmetries and generation of solutions for partial differential equations SIGMA, 2007, V.3, 2007, 019, 14 pages.

[67] Tychynin V. and Rasin O., Nonlocal Symmetry and Generating Solutions for the Inhomogeneous Burgers Equation, in Proceedings of the Fifth International Conference "Symmetry in Nonlinear Mathematical Physics", Proceedings of Institute of Mathematics, Kyiv, 2004, V.50, Part 1, 277-281.

[68] Weisner L, Generating functions for Hermite functions, Canad. J. Math, 1959, V.11, 141-147.

[69] Widder D.V., The heat equation, New-York, Academic Press, 1975.

[70] Yang Q.-j., Chen X.-Z., Zheng K.-j. and Pan Z.-L., Similarity solutions to three-dimensional nonlinear diffusion equations, J. Phys. A: Math.Gen., 1990, V.23, 265-269.

[71] C.M. Yung, K. Verburg and P. Baveye, Group classification and symmetry reductions of the non-linear diffusion-convection equation $u_{t}=\left(D(u) u_{x}\right)_{x}-K^{\prime}(u) u_{x}$, Int. J. Non-Lin. Mech., V.29, 1994, 273-278.

[72] Zel'dovich Ya.B. and Kompaneets A.S., On the theory of propagation of heat with the heat conductivity depending upon the temperature, Collection in Honor of the Seventieth Birthday of Academician A. F. Ioffe [in Russian], Izdat. Akad. Nauk SSSR, Moscow, 1950, 61-71.

[73] Zhdanov R.Z. and Lahno V.I., Conditional symmetry of a porous medium equation, Physica D, V.122, 1998, 178-186.

[74] Zwillinger D., Handbook of differential equations, San Diego, Academic Press, 1989. 


\section{Appendix A. Group classification of diffusion-convection equations}

Table 14. Case of $\forall A(u), g=1$

\begin{tabular}{|l|c|c|c|l|}
\hline $\mathrm{N}$ & $B(u)$ & $f(x)$ & $h(x)$ & \multicolumn{1}{|c|}{ Basis of $\mathrm{A}^{\max }$} \\
\hline 1 & $\forall$ & $\forall$ & $\forall$ & $\partial_{t}$ \\
$2 \mathrm{a}$ & $\forall$ & $e^{p x}$ & 1 & $\partial_{t}, p t \partial_{t}+\partial_{x}$ \\
$2 \mathrm{a}^{\prime}$ & $\forall$ & $|x|^{p}$ & $x^{-1}$ & $\partial_{t},(p+2) t \partial_{t}+x \partial_{x}$ \\
$2 \mathrm{~b}$ & 1 & $e^{x}$ & $e^{x}+\beta$ & $\partial_{t}, e^{-t}\left(\partial_{t}-\partial_{x}\right)$ \\
$2 \mathrm{c}$ & 1 & $|x|^{p}$ & $x|x|^{p}+\beta x^{-1}$ & $\partial_{t}, e^{-(p+2) t}\left(\partial_{t}-x \partial_{x}\right)$ \\
3 & 1 & $x^{-2}$ & $x^{-1} \ln |x|$ & $\partial_{t}, e^{-t} x \partial_{x}$ \\
4 & 0 & 1 & 1 & $\partial_{t}, \partial_{x}, 2 t \partial_{t}+x \partial_{x}$ \\
\hline
\end{tabular}

Here $p \in\{0,1\} \bmod G_{1}^{\sim}$ in case $2 ; p \neq-2$ in case $2 \mathrm{c} ; \beta \in\{0, \pm 1\}$ in case $2 \mathrm{~b}$. Additional equivalence transformations:

1. $2 \mathrm{a}(p=0, B=1) \rightarrow 2 \mathrm{a}(p=0, B=0): \tilde{t}=t, \tilde{x}=x+t, \tilde{u}=u$;

$1^{\prime} \cdot 2 \mathrm{a}^{\prime}(p=-2, B=1) \rightarrow 2 \mathrm{a}^{\prime}(p=-2, B=0): \tilde{t}=t, \tilde{x}=x e^{t}, \tilde{u}=u$;

2. $2 \mathrm{~b} \rightarrow 2 \mathrm{a}(B=\beta, p=1): \tilde{t}=e^{t}, \tilde{x}=x+t, \tilde{u}=u$;

3. $2 \mathrm{c}(p \neq-2) \rightarrow 2^{\prime} \mathrm{a}(p \neq-2): \quad \tilde{t}=\left(e^{(p+2) t}-1\right) /(p+2), \tilde{x}=x e^{t}, \tilde{u}=u$.

Table 15. Case of $A(u)=e^{\mu u}$

\begin{tabular}{|l|c|c|c|c|l|}
\hline $\mathrm{N}$ & $B(u)$ & $f(x)$ & $g(x)$ & $h(x)$ & \multicolumn{1}{|c|}{ Basis of $\mathrm{A}^{\max }$} \\
\hline 1 & 0 & $\forall$ & 1 & 1 & $\partial_{t}, t \partial_{t}-\partial_{u}$ \\
2 & $e^{\nu u}$ & $|x|^{p}$ & 1 & $|x|^{q}$ & $\left.\partial_{t}, p \mu-p \nu-2 \nu-q \mu+\mu\right) t \partial_{t}$ \\
& & & & & $+\left((\mu-\nu) x \partial_{x}+(q+1) \partial_{u}\right.$ \\
$2^{*}$ & $e^{\nu u}$ & $e^{p x}$ & 1 & $\varepsilon e^{x}$ & $\partial_{t},(p \mu-p \nu-\mu) t \partial_{t}+(\mu-\nu) \partial_{x}+\partial_{u}$ \\
3 & $u e^{u}$ & $e^{p x^{2}+q x}$ & $e^{p x^{2}}$ & $e^{p x^{2}}$ & $\partial_{t},(2 p+q) t \partial_{t}+\partial_{x}-2 p \partial_{u}$ \\
4 & $e^{\nu u}+\varkappa$ & 1 & 1 & 1 & $\partial_{t}, \partial_{x},(\mu-2 \nu) t \partial_{t}+((\mu-\nu) x+\nu x t) \partial_{x}+\partial_{u}$ \\
5 & $u$ & 1 & 1 & 1 & $\partial_{t}, \partial_{x}, t \partial_{t}+(x-t) \partial_{x}+\partial_{u}$ \\
$6 \mathrm{a}$ & 0 & $f^{1}(x)$ & 1 & 1 & $\partial_{t}, t \partial_{t}-\partial_{u}, \alpha t \partial_{t}+\left(\beta x^{2}+\gamma_{1} x+\gamma_{0}\right) \partial_{x}+\beta x \partial_{u}$ \\
$6 \mathrm{~b}$ & 1 & $|x|^{p}$ & 1 & $\varepsilon x|x|^{p}$ & $\partial_{t}, x \partial_{x}+(p+2) \partial_{u}, e^{-\varepsilon(p+2) t}\left(\partial_{t}-\varepsilon x \partial_{x}\right)$ \\
$6 \mathrm{~b}^{*}$ & 1 & $e^{x}$ & 1 & $\varepsilon e^{x}$ & $\partial_{t}, \partial_{x}+\partial_{u}, e^{-\varepsilon t}\left(\partial_{t}-\varepsilon \partial_{x}\right)$ \\
$6 \mathrm{c}$ & 1 & $x^{-2}$ & 1 & $\varepsilon x^{-1}$ & $\partial_{t}, x \partial_{x}, t \partial_{t}-\varepsilon t x \partial_{x}-\partial_{u}$ \\
$7 \mathrm{a}$ & 0 & 1 & 1 & 1 & $\partial_{t}, t \partial_{t}-\partial_{u}, 2 t \partial_{t}+x \partial_{x}, \partial_{x}$ \\
$7 \mathrm{~b}$ & 1 & 1 & 1 & 1 & $\partial_{t}, \partial_{x}, t \partial_{t}-t \partial_{x}-\partial_{u}, 2 t \partial_{t}+(x-t) \partial_{x}$ \\
$7 \mathrm{c}$ & 1 & 1 & 1 & $\varepsilon x$ & $\partial_{t}, x \partial_{x}+2 \partial_{u}, e^{-\varepsilon t} \partial_{x}, e^{-2 \varepsilon t}\left(\partial_{t}-\varepsilon x \partial_{x}\right)$ \\
$7 \mathrm{~d}$ & 0 & $x^{-3}$ & 1 & 1 & $\partial_{t}, t \partial_{t}-\partial_{u}, x \partial_{x}-\partial_{u}, x^{2} \partial_{x}+x \partial_{u}$ \\
$7 \mathrm{e}$ & 1 & $x^{-3}$ & 1 & $x^{-2}$ & $\partial_{t}, x \partial_{x}-\partial_{u}, e^{t}\left(\partial_{t}-x \partial_{x}\right), e^{t}\left(x^{2} \partial_{x}+x \partial_{u}\right)$ \\
\hline
\end{tabular}

Here $(\mu, \nu) \in\{(0,1),(1, \nu)\}, \nu \neq \mu$ in cases $2,2^{*}$ and $4 ; \mu=1$ and $\nu \neq 1$ in the other cases; $q \neq-1$ in case $2^{*}$ (otherwise it is subcase of the case $1.2 \mathrm{a}^{\prime}$ ); $\varepsilon= \pm 1$ in cases $2,6 \mathrm{~b}-6 \mathrm{c}$ and $7 \mathrm{e}$; $p \notin\{-3,-2,0\}$ in case $6 \mathrm{~b} ; \alpha, \beta, \gamma_{1}, \gamma_{0}=$ const and

$$
f^{1}(x)=\exp \left\{\int \frac{-3 \beta x-2 \gamma_{1}+\alpha}{\beta x^{2}+\gamma_{1} x+\gamma_{0}} d x\right\} .
$$

Case $2(q=-1)$ is a subcase of case $1.2 \mathrm{a}^{\prime}$. Additional equivalence transformations:

1. $4(\varkappa \neq 0) \rightarrow 4(\varkappa=0): \quad \tilde{t}=t, \tilde{x}=x+\varkappa t, \tilde{u}=u$;

2. $6 \mathrm{~b} \rightarrow 6 \mathrm{a}\left(\beta=\gamma_{0}=0, \alpha=(p+2) \gamma_{1}\right): \quad \tilde{t}=\left(e^{\varepsilon(p+2) t}-1\right) /(\varepsilon(p+2)), \tilde{x}=x e^{\varepsilon t}, \tilde{u}=u$;

3. $6 \mathrm{~b}^{*} \rightarrow 6 \mathrm{a}\left(\beta=\gamma_{1}=0, \alpha=\gamma_{0}\right): \tilde{t}=e^{\varepsilon t} / \varepsilon, \tilde{x}=x+\varepsilon t, \tilde{u}=u$; 
4. $6 \mathrm{c} \rightarrow 6 \mathrm{a}\left(\beta=\gamma_{0}=\alpha=0\right): \tilde{t}=t, \tilde{x}=x e^{\varepsilon t}, \tilde{u}=u$;

5. $7 \mathrm{~b} \rightarrow 7 \mathrm{a}: \quad \tilde{t}=t, \tilde{x}=x+t, \tilde{u}=u$;

6. $7 \mathrm{c} \rightarrow 7 \mathrm{a}: \quad \tilde{t}=e^{2 \varepsilon t} /(2 \varepsilon), \tilde{x}=x e^{\varepsilon t}, \tilde{u}=u$;

7. $7 \mathrm{~d} \rightarrow 7 \mathrm{a}: \quad \tilde{t}=t \operatorname{sign} x, \tilde{x}=1 / x, \tilde{u}=u-\ln |x|$;

8. $7 \mathrm{e} \rightarrow 7 \mathrm{a}: \quad \tilde{t}=\left(e^{2 t} t \operatorname{sign} x\right) / 2, \tilde{x}=e^{-t} / x, \tilde{u}=u-t-\ln |x|$.

Table 16. Case of $A(u)=|u|^{\mu}$

\begin{tabular}{|c|c|c|c|c|c|c|}
\hline $\mathrm{N}$ & $\mu$ & $B(u)$ & $f(x)$ & $g(x)$ & $h(x)$ & Basis of $A^{\max }$ \\
\hline 1 & $\forall$ & 0 & $\forall$ & 1 & 1 & $\partial_{t}, \mu t \partial_{t}-u \partial_{u}$ \\
\hline 2 & $\forall$ & $|u|^{\nu}$ & $|x|^{p}$ & 1 & $|x|^{q}$ & $\begin{array}{l}\partial_{t},(\mu+p \mu-q \mu-p \nu-2 \nu) t \partial_{t} \\
+(\mu-\nu) x \partial_{x}+(q+1) u \partial_{u}\end{array}$ \\
\hline $2^{*}$ & $\forall$ & $|u|^{\nu}$ & $e^{p x}$ & 1 & $\varepsilon e^{x}$ & $\partial_{t},(p \mu-p \nu-\mu) t \partial_{t}+(\mu-\nu) \partial_{x}+u \partial_{u}$ \\
\hline 3 & $\forall$ & $|u|^{\mu} \ln |u|$ & $e^{p x^{2}+q x}$ & $e^{p x^{2}}$ & $e^{p x^{2}}$ & $\partial_{t},(2 \mu p+q) t \partial_{t}+\partial_{x}-2 p u \partial_{u}$ \\
\hline 4 & $\forall$ & 1 & $f^{2}$ & 1 & $\varepsilon x f^{2}$ & $\partial_{t}$ \\
\hline 5 & 0 & $\forall$ & $e^{p x^{2}}$ & $e^{p x^{2}}$ & $e^{p x^{2}}$ & $\begin{array}{l}e^{\varepsilon t}\left(\partial_{t}-\varepsilon\left((\mu+1) \beta x^{2}+x\right) \partial_{x}-\varepsilon \beta x u \partial_{u}\right) \\
\partial_{t}, e^{-2 p t} \partial_{x}\end{array}$ \\
\hline 6 & 0 & $\forall$ & $e^{x+\gamma e^{x}}$ & $e^{\gamma e^{x}}$ & $e^{\gamma e^{x}}$ & $\partial_{t}, e^{-\gamma t}\left(\partial_{t}-\gamma \partial_{x}\right)$ \\
\hline 7 & 0 & $u$ & $e^{p x^{2}+x}$ & $e^{p x^{2}}$ & $e^{p x^{2}}$ & $\partial_{t}, t \partial_{t}+\partial_{x}-2 p \partial_{u}$ \\
\hline 8 & $\forall$ & $|u|^{\nu}+\varkappa$ & 1 & 1 & 1 & $\partial_{t}, \partial_{x}$ \\
\hline 9 & $\forall$ & $\ln |u|$ & 1 & 1 & 1 & $\begin{array}{l}(\mu-2 \nu) t \partial_{t}+((\mu-\nu) x+\nu \varkappa t) \partial_{x}+u \partial_{u} \\
\partial_{t}, \partial_{x}, \mu t \partial_{t}+(\mu x-t) \partial_{x}+u \partial_{u}\end{array}$ \\
\hline 10 & 0 & $u$ & $e^{p x^{2}}$ & $e^{p x^{2}}$ & $e^{p x^{2}}$ & $\partial_{t}, e^{-2 p t} \partial_{x}, \partial_{x}-2 p \partial_{u}$ \\
\hline 11 & 0 & $\ln |u|$ & $e^{p x^{2}}$ & $e^{p x^{2}}$ & $e^{p x^{2}}$ & $\partial_{t}, e^{-2 p t} \partial_{x}, \partial_{x}-2 p u \partial_{u}$ \\
\hline $12 \mathrm{a}$ & $\forall$ & 0 & $f^{3}$ & 1 & 1 & $\partial_{t}, \mu t \partial_{t}-u \partial_{u}, \alpha t \partial_{t}$ \\
\hline $12 \mathrm{~b}$ & $\forall$ & 1 & $|x|^{p}$ & 1 & $\varepsilon x|x|^{p}$ & $\begin{array}{l}+\left((\mu+1) \beta x^{2}+\gamma_{1} x+\gamma_{0}\right) \partial_{x}+\beta x u \partial_{u} \\
\partial_{t}, \mu x \partial_{x}+(p+2) u \partial_{u} \\
e^{-\varepsilon(p+2) t}\left(\partial_{t}-\varepsilon x \partial_{x}\right)\end{array}$ \\
\hline $12 b^{*}$ & $\neq-1$ & 1 & $e^{x}$ & 1 & $\varepsilon e^{x}$ & $\partial_{t}, \mu \partial_{x}+u \partial_{u}, e^{-\varepsilon t}\left(\partial_{t}-\varepsilon \partial_{x}\right)$ \\
\hline $12 \mathrm{c}$ & $\neq-2$ & 1 & $x^{-2}$ & 1 & $\varepsilon x^{-1}$ & $\partial_{t}, x \partial_{x}, \mu t \partial_{t}-\varepsilon \mu t x \partial_{x}-u \partial_{u}$ \\
\hline 13 & $-\frac{6}{5}$ & 1 & $x^{2}$ & 1 & $x^{2}$ & $\partial_{t}, 2 t \partial_{t}+2 x \partial_{x}-5 u \partial_{u}$ \\
\hline $14 \mathrm{a}$ & $\neq-\frac{4}{2}$ & 0 & 1 & 1 & 1 & $\begin{array}{l}t^{2} \partial_{t}+\left(2 t x+x^{2}\right) \partial_{x}-5(t+x) u \partial_{u} \\
\partial_{t}, \mu t \partial_{t}-u \partial_{u}, \partial_{x}, 2 t \partial_{t}+x \partial_{x}\end{array}$ \\
\hline $14 \mathrm{~b}$ & $\begin{aligned} & 3 \\
& \neq-\frac{4}{3}\end{aligned}$ & 1 & 1 & 1 & 1 & $\partial_{t}, \mu t \partial_{t}-\mu t \partial_{x}-u \partial_{u}, \partial_{x}$ \\
\hline & & & & & & $2 t \partial_{t}+(x-t) \partial_{x}$ \\
\hline $14 \mathrm{c}$ & $\neq-\frac{4}{3}$ & 1 & 1 & 1 & $\varepsilon x$ & $\begin{array}{l}\partial_{t}, \mu x \partial_{x}+2 u \partial_{u}, e^{-\varepsilon t} \partial_{x} \\
e^{-2 \varepsilon t}\left(\partial_{t}-\varepsilon x \partial_{x}\right)\end{array}$ \\
\hline
\end{tabular}


Table 16. Continuation

\begin{tabular}{|c|c|c|c|c|c|c|}
\hline $\mathrm{N}$ & $\mu$ & $B(u)$ & $f(x)$ & $g(x)$ & $h(x)$ & Basis of $\mathrm{A}^{\max }$ \\
\hline $14 \mathrm{~d}$ & $\begin{array}{l}\neq-\frac{4}{3} \\
-1\end{array}$ & 0 & $|x|^{s(\mu)}$ & 1 & 1 & $\begin{array}{l}\partial_{t}, \mu t \partial_{t}-u \partial_{u},(\mu+2) t \partial_{t}-(\mu+1) x \partial_{x} \\
(\mu+1) x^{2} \partial_{x}+x u \partial_{u}\end{array}$ \\
\hline $14 \mathrm{e}$ & $\begin{array}{l}\neq-2 \\
-\frac{4}{3},-1\end{array}$ & 1 & $|x|^{s(\mu)}$ & 1 & $\varepsilon x|x|^{s(\mu)}$ & $\begin{array}{l}\partial_{t}, \mu(\mu+1) x \partial_{x}-(\mu+2) u \partial_{u} \\
e^{\varepsilon \frac{\mu+2}{\mu+1} t}\left(\partial_{t}-\varepsilon x \partial_{x}\right) \\
e^{\varepsilon t}\left((\mu+1) x^{2} \partial_{x}+x u \partial_{u}\right)\end{array}$ \\
\hline $14 \mathrm{f}$ & -1 & 0 & $e^{x}$ & 1 & 1 & $\begin{array}{l}\partial_{t}, t \partial_{t}+u \partial_{u} \\
\partial_{x}-u \partial_{u}, 2 t \partial_{t}+x \partial_{x}-x u \partial_{u}\end{array}$ \\
\hline $14 \mathrm{~g}$ & -1 & 1 & $e^{x}$ & 1 & $\varepsilon e^{x}$ & $\begin{array}{l}\partial_{t}, \partial_{x}-u \partial_{u}, e^{-\varepsilon t}\left(\partial_{t}-\varepsilon \partial_{x}\right) \\
(x+\varepsilon t-2) \partial_{x}-(x+\varepsilon t) u \partial_{u}\end{array}$ \\
\hline $14 \mathrm{~h}$ & -2 & 1 & $x^{-2}$ & 1 & $\varepsilon x^{-1}$ & $\begin{array}{l}\partial_{t}, x \partial_{x}, 2 t \partial_{t}-2 \varepsilon t x \partial_{x}+u \partial_{u} \\
e^{\varepsilon t}\left(x^{2} \partial_{x}-x u \partial_{u}\right)\end{array}$ \\
\hline $15 \mathrm{a}$ & $-\frac{4}{3}$ & 0 & 1 & 1 & 1 & $\begin{array}{l}\partial_{t}, 4 t \partial_{t}+3 u \partial_{u}, \partial_{x}, 2 t \partial_{t}+x \partial_{x} \\
x^{2} \partial_{x}-3 x u \partial_{u}\end{array}$ \\
\hline $15 \mathrm{~b}$ & $-\frac{4}{3}$ & 1 & 1 & 1 & 1 & $\begin{array}{l}\partial_{t}, 4 t \partial_{t}+4 x \partial_{x}-3 u \partial_{u}, 2 t \partial_{t}+(x-t) \partial_{x} \\
\partial_{x},(x+t)^{2} \partial_{x}-3(x+t) u \partial_{u}\end{array}$ \\
\hline $15 \mathrm{c}$ & $-\frac{4}{3}$ & 1 & 1 & 1 & $\varepsilon x$ & $\begin{array}{l}\partial_{t}, 2 x \partial_{x}-3 u \partial_{u}, \\
e^{-\varepsilon t} \partial_{x} \\
e^{-2 \varepsilon t}\left(\partial_{t}-\varepsilon x \partial_{x}\right), e^{\varepsilon t}\left(x^{2} \partial_{x}-3 x u \partial_{u}\right)\end{array}$ \\
\hline 16 & 0 & $u$ & 1 & 1 & 1 & $\begin{array}{l}\partial_{t}, \partial_{x}, t \partial_{x}-\partial_{u}, 2 t \partial_{t}+x \partial_{x}-u \partial_{u} \\
t^{2} \partial_{t}+t x \partial_{x}-(t u+x) \partial_{u}\end{array}$ \\
\hline
\end{tabular}

Here $s(\mu)=-\frac{3 \mu+4}{\mu+1}, \nu \neq \mu ; \varepsilon= \pm 1 ; q \neq-1$ in case $2^{*}$ (otherwise it is subcase of the case $1.2 \mathrm{a}^{\prime}$ ); $p \neq-2,-(3 \mu+4) /(\mu+1)$ in case $12 \mathrm{c} ; \alpha, \beta, \gamma_{1}, \gamma_{0}=$ const, and

$$
f^{2}(x)=\exp \left\{\int \frac{-(3 \mu+4) \beta x-3}{(\mu+1) \beta x^{2}+x} d x\right\}, \quad f^{3}(x)=\exp \left\{\int \frac{-(3 \mu+4) \beta x-2 \gamma_{1}+\alpha}{(\mu+1) \beta x^{2}+\gamma_{1} x+\gamma_{0}} d x\right\} .
$$

Additional equivalence transformations:

1. $8(\varkappa \neq 0) \rightarrow 8(\varkappa=0): \tilde{t}=t, \tilde{x}=x+\varkappa t, \tilde{u}=u$;

2. $12 \mathrm{~b} \rightarrow 12 \mathrm{a}\left(\beta=\gamma_{0}=\alpha=0\right), 14 \mathrm{e} \rightarrow 14 \mathrm{a}: \tilde{t}=\left(e^{(p+2) t}-1\right) /(p+2), \tilde{x}=x e^{t}, \tilde{u}=u$;

3. $12 \mathrm{~b}^{*} \rightarrow 12 \mathrm{a}\left(\beta=\gamma_{1}=0, \alpha=\gamma_{0}\right): \quad \tilde{t}=\left(e^{\varepsilon(p+2) t}-1\right) /(\varepsilon(p+2)), \tilde{x}=x e^{\varepsilon t}, \tilde{u}=u$;

4. $12 \mathrm{c} \rightarrow 12 \mathrm{a}\left(\beta=\gamma_{0}=\alpha=0\right), 14 \mathrm{~h} \rightarrow 14 \mathrm{a}: \tilde{t}=t, \tilde{x}=x e^{\varepsilon t}, \tilde{u}=u$;

5. $14 \mathrm{~b} \rightarrow 14 \mathrm{a}, 15 \mathrm{~b} \rightarrow 15 \mathrm{a}: \tilde{t}=t, \tilde{x}=x-t, \tilde{u}=u$;

6. $14 \mathrm{c} \rightarrow 14 \mathrm{a}, 15 \mathrm{c} \rightarrow 15 \mathrm{a}: \tilde{t}=e^{2 \varepsilon t} /(2 \varepsilon), \tilde{x}=x e^{\varepsilon t}, \tilde{u}=u$;

7. $14 \mathrm{~d} \rightarrow 14 \mathrm{a}: \tilde{t}=t, \tilde{x}=-1 / x, \tilde{u}=|x|^{-\frac{1}{1+\mu}} u$;

8. $14 \mathrm{f} \rightarrow 14 \mathrm{a}(\mu=-1): \tilde{t}=t, \tilde{x}=x, \tilde{u}=e^{x} u$.

9. $14 \mathrm{~g} \rightarrow 14 \mathrm{a}(\mu=-1): \tilde{t}=e^{\varepsilon t} / \varepsilon, \tilde{x}=x+\varepsilon t, \tilde{u}=e^{x+\varepsilon t} u$;

Institute of Mathematics of NAS of Ukraine, 3 Tereshchenkivska Str., 01601 Kyiv4, UKRaine

E-mail address: ivanova@imath.kiev.ua 